\title{
Cluster mutation-periodic quivers and associated Laurent sequences
}

\author{
Allan P. Fordy • Bethany R. Marsh
}

Received: 10 February 2010 / Accepted: 18 October 2010 / Published online: 4 November 2010

(C) Springer Science+Business Media, LLC 2010

\begin{abstract}
We consider quivers/skew-symmetric matrices under the action of mutation (in the cluster algebra sense). We classify those which are isomorphic to their own mutation via a cycle permuting all the vertices, and give families of quivers which have higher periodicity.

The periodicity means that sequences given by recurrence relations arise in a natural way from the associated cluster algebras. We present a number of interesting new families of nonlinear recurrences, necessarily with the Laurent property, of both the real line and the plane, containing integrable maps as special cases. In particular, we show that some of these recurrences can be linearised and, with certain initial conditions, give integer sequences which contain all solutions of some particular Pell equations. We extend our construction to include recurrences with parameters, giving an explanation of some observations made by Gale.

Finally, we point out a connection between quivers which arise in our classification and those arising in the context of quiver gauge theories.
\end{abstract}

Keywords Cluster algebra - Quiver mutation · Periodic quiver · Somos sequence · Integer sequences · Pell's equation · Laurent phenomenon · Integrable map · Linearisation $\cdot$ Seiberg duality $\cdot$ Supersymmetric quiver gauge theory

\section{Introduction}

Our main motivation for this work is the connection between cluster algebras and integer sequences which are Laurent polynomials in their initial terms [8]. A key

A.P. Fordy · B.R. Marsh $凶)$

School of Mathematics, University of Leeds, Leeds LS2 9JT, UK

e-mail: B.R.marsh@leeds.ac.uk

A.P. Fordy

e-mail: allan@maths.leeds.ac.uk 


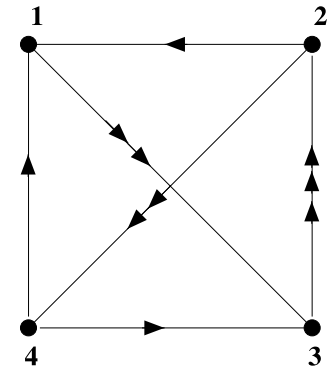

(a) The Somos 4 quiver, $S_{4}$.

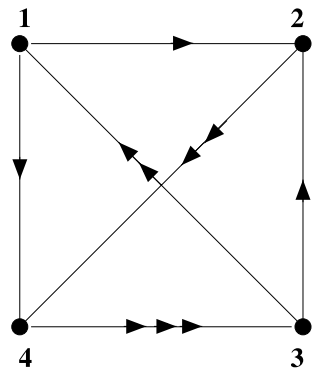

(b) Mutation of $S_{4}$ at 1 .

Fig. 1 The Somos 4 quiver and its mutation at 1

example of this is the Somos 4 sequence, which is given by the following recurrence:

$$
x_{n} x_{n+4}=x_{n+1} x_{n+3}+x_{n+2}^{2} .
$$

This formula, with appropriate relabelling of the variables, coincides with the cluster exchange relation [7] (recalled below; see Sect. 8) associated with the vertex 1 in the quiver $S_{4}$ of Fig. 1(a). Mutation of $S_{4}$ at 1 (as in [7]; see Definition 2.1 below) gives the quiver shown in Fig. 1(b) and transforms the cluster $\left(x_{1}, x_{2}, x_{3}, x_{4}\right)$ into $\left(\tilde{x}_{1}, x_{2}, x_{3}, x_{4}\right)$, where $\tilde{x}_{1}$ is given by

$$
x_{1} \tilde{x}_{1}=x_{2} x_{4}+x_{3}^{2} .
$$

Remarkably, after this complicated operation of mutation on the quiver, the result is a simple rotation, corresponding to the relabelling of indices $(1,2,3,4) \mapsto(4,1,2,3)$. Therefore, a mutation of the new quiver at 2 gives the same formula for the exchange relation (up to a relabelling). It is this simple property that allows us to think of an infinite sequence of such mutations as iteration of recurrence (1).

In this paper, we classify quivers with this property. In this way we obtain a classification of maps which could be said to be 'of Somos type'. In fact we consider a more general type of "mutation periodicity", which corresponds to Somos type sequences of higher dimensional spaces.

It is interesting to note that many of the quivers which have occurred in the theoretical physics literature concerning supersymmetric quiver gauge theories are particular examples from our classification; see for example [5, §4]. We speculate that some of our other examples may be of interest in that context.

We now describe the contents of the article in more detail. In Sect. 2, we recall matrix and quiver mutation from [7], and introduce the notion of periodicity we are considering. It turns out to be easier to classify periodic quivers if we assume that certain vertices are sinks; we call such quivers sink-type. In Sect. 3, we classify the sink-type quivers of period 1 as nonnegative integer combinations of a family of primitive quivers. In Sect. 4, we do the same for sink-type period 2 quivers, and in Sect. 5 we classify the sink-type quivers of arbitrary period.

In Sect. 6, we give a complete classification of all period 1 quivers (without the sink assumption), and give some examples. It turns out the arbitrary period 1 quivers 
can be described in terms of the primitives with $N$ nodes, together with the primitives for quivers with $N^{\prime}$ nodes for all $N^{\prime}$ less than $N$ of the same parity (Theorem 6.6).

In Sect. 7, we classify quivers of period 2 with at most five nodes. These descriptions indicate that a full classification for higher period is likely to be significantly more complex than the classification of period 1 quivers. However, it is possible to construct a large family of period 2 (not of sink-type) quivers, which we present in Sect. 7.4.

In Sect. 8, we describe the recurrences that can be associated to period 1 and period 2 quivers via Fomin-Zelevinsky cluster mutation. The nature of the cluster exchange relation means that the recurrences we have associated to periodic quivers are in general nonlinear. However, in Sect. 9, we show that the recurrences associated to period 1 primitives can be linearised. This allows us to conclude in Sect. 9 that certain simple linear combinations of subsequences of the first primitive period 1 quiver (for arbitrarily many nodes) provide all the solutions to an associated Pell equation.

In Sect. 10 we extend our construction of mutation periodic quivers to include quivers with frozen cluster variables, thus enabling the introduction of parameters into the corresponding recurrences. As a result, we give an explanation of some observations made by Gale in [13].

In Sect. 11, we give an indication of the connections with supersymmetric quiver gauge theories.

In Sect. 12, we present our final conclusions. The last section is an appendix to Sect. 9.

\section{The periodicity property}

We consider quivers with no 1-cycles or 2-cycles (i.e. the quivers on which cluster mutation is defined). Any 2-cycles which arise through operations on the quiver will be cancelled. The vertices of $Q$ will be assumed to lie on the vertices of a regular $N$-sided polygon, labelled $1,2, \ldots, N$ in clockwise order.

In the usual way, we shall identify a quiver $Q$, with $N$ nodes, with the unique skew-symmetric $N \times N$ matrix $B_{Q}$ with $\left(B_{Q}\right)_{i j}$ given by the number of arrows from $i$ to $j$ minus the number of arrows from $j$ to $i$. We next recall the definition of quiver mutation [7].

Definition 2.1 (Quiver mutation) Given a quiver $Q$ we can mutate at any of its nodes. The mutation of $Q$ at node $k$, denoted by $\mu_{k} Q$, is constructed (from $Q$ ) as follows:

1. Reverse all arrows which either originate or terminate at node $k$.

2. Suppose that there are $p$ arrows from node $i$ to node $k$ and $q$ arrows from node $k$ to node $j$ (in $Q$ ). Add $p q$ arrows going from node $i$ to node $j$ to any arrows already there.

3. Remove (both arrows of) any two-cycles created in the previous steps.

Note that Step 3 is independent of any choices made in the removal of the twocycles, since the arrows are not labelled. We also note that in Step 2, $p q$ is just the number of paths of length 2 between nodes $i$ and $j$ which pass through node $k$. 
Remark 2.2 (Matrix mutation) Let $B$ and $\tilde{B}$ be the skew-symmetric matrices corresponding to the quivers $Q$ and $\tilde{Q}=\mu_{k} Q$. Let $b_{i j}$ and $\tilde{b}_{i j}$ be the corresponding matrix entries. Then quiver mutation amounts to the following formula

$$
\tilde{b}_{i j}= \begin{cases}-b_{i j} & \text { if } i=k \text { or } j=k, \\ b_{i j}+\frac{1}{2}\left(\left|b_{i k}\right| b_{k j}+b_{i k}\left|b_{k j}\right|\right) & \text { otherwise. }\end{cases}
$$

This is the original formula appearing (in a more general context) in [7].

We number the nodes from 1 to $N$, arranging them equally spaced on a circle (clockwise ascending). We consider the permutation $\rho:(1,2, \ldots, N) \rightarrow$ $(N, 1, \ldots, N-1)$. Such a permutation acts on a quiver $Q$ in such a way that the number of arrows from $i$ to $j$ in $Q$ is the same as the number of arrows from $\rho^{-1}(i)$ to $\rho^{-1}(j)$ in $\rho Q$. Thus the arrows of $Q$ are rotated clockwise while the nodes remain fixed (alternatively, this operation can be interpreted as leaving the arrows fixed whilst the nodes are moved in an anticlockwise direction). We will always fix the positions of the nodes in our diagrams.

Note that the action $Q \mapsto \rho Q$ corresponds to the conjugation $B_{Q} \mapsto \rho B_{Q} \rho^{-1}$, where

$$
\rho=\left(\begin{array}{cccc}
0 & \cdots & \cdots & 1 \\
1 & 0 & & \vdots \\
& \ddots & \ddots & \vdots \\
& & 1 & 0
\end{array}\right)
$$

(we will use the notation $\rho$ for both the permutation and corresponding matrix).

We consider a sequence of mutations, starting at node 1 , followed by node 2 , and so on. Mutation at node 1 of a quiver $Q(1)$ will produce a second quiver $Q(2)$. The mutation at node 2 will therefore be of quiver $Q(2)$, giving rise to quiver $Q(3)$ and so on.

Definition 2.3 We will say that a quiver $Q$ has period $m$ if it satisfies $Q(m+1)=$ $\rho^{m} Q(1)$, with the mutation sequence depicted by

$$
Q=Q(1) \stackrel{\mu_{1}}{\longrightarrow} Q(2) \stackrel{\mu_{2}}{\longrightarrow} \cdots \stackrel{\mu_{m-1}}{\longrightarrow} Q(m) \stackrel{\mu_{m}}{\longrightarrow} Q(m+1)=\rho^{m} Q(1) .
$$

We call the above sequence of quivers the periodic chain associated to $Q$.

Note that permutations other than $\rho^{m}$ could be used here, but we do not consider them in this article. If $m$ is minimal in the above, we say that $Q$ is strictly of period $m$. Also note that each of the quivers $Q(1), \ldots, Q(m)$ is of period $m$ (with a renumbering of the vertices), if $Q$ is.

Recall that a node $i$ of a quiver $Q$ is said to be a sink if all arrows incident with $i$ end at $i$, and is said to be a source if all arrows incident with $i$ start at $i$.

Remark 2.4 (Admissible sequences) Recall that an admissible sequence of sinks in an acyclic quiver $Q$ is a total ordering $v_{1}, v_{2}, \ldots, v_{N}$ of its vertices such that $v_{1}$ is a sink 
in $Q$ and $v_{i}$ is a sink in $\mu_{v_{i-1}} \mu_{v_{i-2}} \cdots \mu_{v_{1}}(Q)$ for $i=2,3, \ldots, N$. Such a sequence always has the property that $\mu_{v_{N}} \mu_{v_{N-1}} \cdots \mu_{v_{1}}(Q)=Q[1, \S 5.1]$. This notion is of importance in the representation theory of quivers.

We note that if any (not necessarily acyclic) quiver $Q$ has period 1 in our sense, then $\mu_{1} Q=\rho Q$. It follows that $\mu_{N} \mu_{N-1} \cdots \mu_{1} Q=Q$. Thus any period 1 quiver has a property which can be regarded as a generalisation of the notion of existence of an admissible sequence of sinks. In fact, higher period quivers also possess this property provided the period divides the number of vertices.

\section{Period 1 quivers}

We now introduce a finite set of particularly simple quivers of period 1, which we shall call the period 1 primitives. Remarkably, it will later be seen that in a certain sense they form a "basis" for the set of all quivers of period 1. We shall also later see that period $m$ primitives can be defined as certain sub-quivers of the period 1 primitives.

Definition 3.1 (Period 1 sink-type quivers) A quiver $Q$ is said to be a period 1 sinktype quiver if it is of period 1 and node 1 of $Q$ is a sink.

Definition 3.2 (Skew-rotation) We shall refer to the matrix

$$
\tau=\left(\begin{array}{cccc}
0 & \cdots & \cdots & -1 \\
1 & 0 & & \vdots \\
& \ddots & \ddots & \vdots \\
& & 1 & 0
\end{array}\right)
$$

as a skew-rotation.

Lemma 3.3 (Period 1 sink-type equation) A quiver $Q$ with a sink at 1 is period 1 if and only if $\tau B_{Q} \tau^{-1}=B_{Q}$.

Proof If node 1 of $Q$ is a sink, there are no paths of length 2 through it, and the second part of Definition 2.1 is void. Reversal of the arrows at node 1 can be done through a simple conjugation of the matrix $B_{Q}$ :

$$
\mu_{1} B_{Q}=D_{1} B_{Q} D_{1}, \quad \text { where } D_{1}=\operatorname{diag}(-1,1, \ldots, 1) .
$$

Equating this to $\rho B_{Q} \rho^{-1}$ leads to the equation $\tau B_{Q} \tau^{-1}=B_{Q}$ as required, noting that

$$
\tau=D_{1} \rho
$$

The map $M \mapsto \tau M \tau^{-1}$ simultaneously cyclically permutes the rows and columns of $M$ (up to a sign), while $\tau^{N}=-I_{N}$, hence $\tau$ has order $N$. This gives us a method for building period 1 matrices: we sum over $\tau$-orbits. 
The period 1 primitives $P_{N}^{(k)}$ We consider a quiver with just a single arrow from $N-k+1$ to 1 , represented by the skew-symmetric matrix $R_{N}^{(k)}$ with $\left(R_{N}^{(k)}\right)_{N-k+1,1}=$ $1,\left(R_{N}^{(k)}\right)_{1, N-k+1}=-1$ and $\left(R_{N}^{(k)}\right)_{i j}=0$ otherwise.

We define skew-symmetric matrices $B_{N}^{(k)}$ as follows:

$$
B_{N}^{(k)}= \begin{cases}\sum_{i=0}^{N-1} \tau^{i} R_{N}^{(k)} \tau^{-i}, & \text { if } N=2 r+1 \text { and } 1 \leq k \leq r, \\ \sum_{i=0}^{r-1} \tau^{i} R_{N}^{(r)} \tau^{-i}, & \text { or if } N=2 r \text { and } 1 \leq k \leq r-1 ;\end{cases}
$$

Let $P_{N}^{(k)}$ denote the quiver corresponding to $B_{N}^{(k)}$. We remark that the geometric action of $\tau$ in the above sum is to rotate the arrow clockwise without change of orientation, except that when the tail of the arrow ends up at node 1 it is reversed. It follows that 1 is a sink in the resulting quiver. Since it is a sum over a $\tau$-orbit, we have $\tau B_{N}^{(k)} \tau^{-1}=B_{N}^{(k)}$, and thus that $P_{N}^{(k)}$ is a period 1 sink-type quiver. In fact, we have the simple description:

$$
B_{N}^{(k)}= \begin{cases}\tau^{k}-\left(\tau^{t}\right)^{k}, & \text { if } N=2 r+1 \text { and } 1 \leq k \leq r, \text { or } N=2 r \text { and } 1 \leq k \leq r-1 \\ \tau^{r}, & \text { if } N=2 r \text { and } k=r,\end{cases}
$$

where $\tau^{t}$ denotes the transpose of $\tau$.

Note that we have restricted to the choice $1 \leq k \leq r$ because when $k>r$, our construction gives nothing new. Firstly, consider the case $N \neq 2 k$. Then $B_{N}^{(N+1-k)}=$ $B_{N}^{(k)}$, because the primitive $B_{N}^{(k)}$ has exactly two arrows ending at 1 : those starting at $k+1$ and at $N+k-1$. Starting with either of these arrows produces the same result. If $N=2 k$, these two arrows are identical, and since $\tau^{k}$ is skew-symmetric, $\tau^{k}-\left(\tau^{t}\right)^{k}=2 \tau^{k}$. The sum over $N=2 k$ terms just goes twice over the sum over $k$ terms.

In this construction we could equally well have chosen node 1 to be a source. We would then have $R_{N}^{(k)} \mapsto-R_{N}^{(k)}, B_{N}^{(k)} \mapsto-B_{N}^{(k)}$ and $P_{N}^{(k)} \mapsto\left(P_{N}^{(k)}\right)^{o p p}$, where $Q^{o p p}$ denotes the opposite quiver of $Q$ (with all arrows reversed). Our original motivation in terms of sequences with the Laurent property is derived through cluster exchange relations, which do not distinguish between a quiver and its opposite, so we consider these as equivalent.

Remark 3.4 We note that each primitive is a disjoint union of cycles or arrows, i.e. quivers whose underlying graph is a union of components which are either of type $A_{2}$ or of type $\widetilde{A}_{m}$ for some $m$.

Figures 2 to 4 show the period 1 primitives we have constructed, for $2 \leq N \leq 6$.

Remark 3.5 (An involution $\iota: Q \mapsto Q^{o p p}$ ) It is easily seen that the following permutation of the nodes is a symmetry of the primitives $P_{N}^{(i)}$ (if we consider $Q$ and $Q^{o p p}$ as equivalent):

$$
\iota:(1,2, \ldots, N) \mapsto(N, N-1, \ldots, 1)
$$




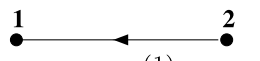

(a) $P_{2}^{(1)}$

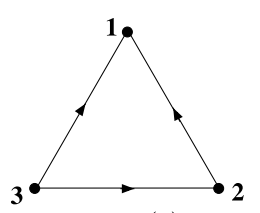

(b) $P_{3}^{(1)}$

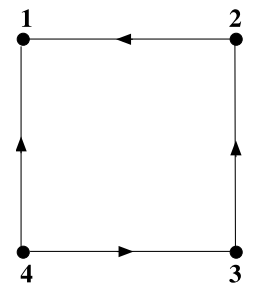

(c) $P_{4}^{(1)}$

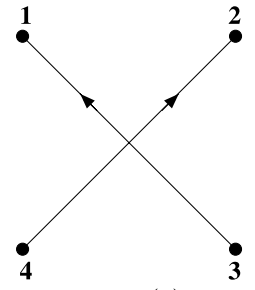

(d) $P_{4}^{(2)}$

Fig. 2 The period 1 primitives for two, three and four nodes

Fig. 3 The period 1 primitives for five nodes

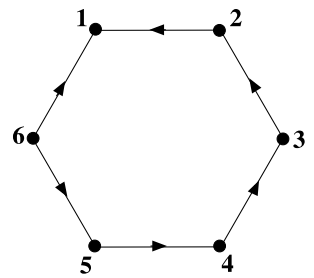

(a) $P_{6}^{(1)}$

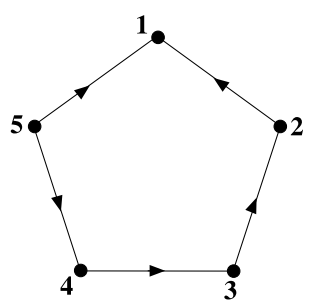

(a) $P_{5}^{(1)}$

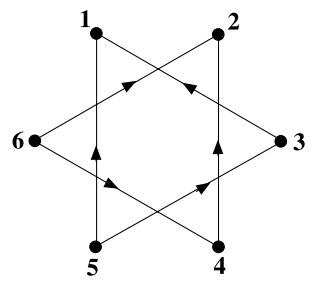

(b) $P_{6}^{(2)}$

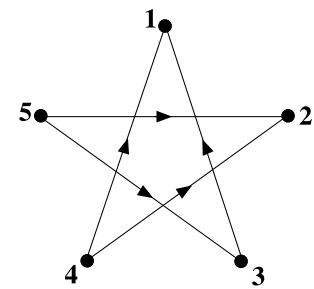

(b) $P_{5}^{(2)}$

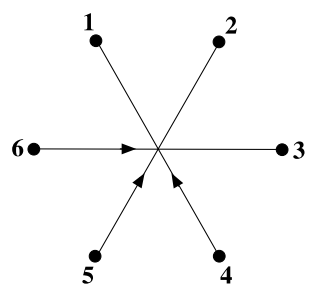

(c) $P_{6}^{(3)}$

Fig. 4 The period 1 primitives for six nodes

In matrix language, this follows from the facts that $\iota R_{N}^{(k)} \iota=-R_{N}^{(k)}$ and $\iota \tau \iota=\tau^{t}$, where

$$
\iota=\left(\begin{array}{llll}
0 & & & 1 \\
& . \cdot & & \\
1 & & & 0
\end{array}\right) .
$$

It is interesting to note that $\rho$ is a Coxeter element in $\Sigma_{N}$ regarded as a Coxeter group, while $\iota$ is the longest element.

We may combine primitives to form more complicated quivers. Consider the sum

$$
P=\sum_{i=1}^{r} m_{i} P_{N}^{(i)}
$$


where $N=2 r$ or $2 r+1$ for $r$ an integer and the $m_{i}$ are arbitrary integers. It is easy to see that the corresponding quiver is a period 1 sink-type quiver whenever $m_{i} \geq 0$ for all $i$. In fact, we have

Proposition 3.6 (Classification of period 1 sink-type quivers) Let $N=2 r$ or $2 r+1$, where $r$ is an integer. Every period 1 sink-type quiver with $N$ nodes has corresponding matrix of the form $B=\sum_{k=1}^{r} m_{k} B_{N}^{(k)}$, where the $m_{k}$ are arbitrary nonnegative integers.

Proof Let $B$ be the matrix of a period 1 sink-type quiver. It remains to show that $B$ is of the form stated. We note that conjugation by $\tau$ permutes the set of summands appearing in the definition (4) of the $B_{N}^{(k)}$, i.e. the elements $\tau^{i} R_{N}^{(k)} \tau^{-i}$ for $0 \leq i \leq$ $N-1$ and $1 \leq k \leq r$ if $N=2 r+1$, for $0 \leq i \leq N-1$ and $1 \leq k \leq r-1$ if $N=2 r$, together with the elements $\tau^{i} R_{N}^{(r)} \tau^{-i}$ for $0 \leq i \leq r-1$ if $N=2 r$. These $\frac{1}{2} N(N-1)$ elements are easily seen to form a basis of the space of real skew-symmetric matrices. By Lemma 3.3, $\tau B \tau^{-1}=B$, so $B$ is a linear combination of the period 1 primitives (which are the orbit sums for the conjugation action of $\tau$ on the above basis), $B=$ $\sum_{k=1}^{r} m_{k} B_{N}^{(k)}$. The support of the $B_{N}^{(k)}$ for $1 \leq k \leq r$ is distinct, so $B_{N-k+1,1}=m_{k}$ for $1 \leq k \leq r$ (where the support of a matrix is the set of positions of its non-zero entries). Hence the $m_{k}$ are integers, as $B$ is an integer matrix. Since $B$ is sink-type, all the $m_{k}$ must be nonnegative.

Note that this means all period 1 sink-type quivers are invariant under $\iota$ in the above sense. We also note that if the $m_{k}$ are taken to be of mixed sign, then $Q$ is no longer periodic without the addition of further "correction" terms. Theorem 6.6 gives these correction terms.

\section{Period 2 quivers}

Period 2 primitives will be defined in a similar way. First, we make the following definition:

Definition 4.1 (Period 2 sink-type quivers) A quiver $Q$ is said to be a period 2 sinktype quiver if it is of period 2, node 1 of $Q(1)=Q$ is a sink, and node 2 of $Q(2)=$ $\mu_{1} Q$ is a sink.

Let $Q$ be a period 2 quiver. Then we have two quivers in our periodic chain (3), $Q(1)$ and $Q(2)=\mu_{1} Q$, with corresponding matrices $B(1), B(2)$. If $Q(1)$ is of sinktype then, since node 1 is a sink in $Q(1)$, the mutation $Q(1) \mapsto \mu_{1} Q(1)=Q(2)$ again only involves the reversal of arrows at node 1 . Similarly, since node 2 is a sink for $Q(2)$, the mutation $Q(2) \mapsto \mu_{2} Q(2)$ only involves the reversal of arrows at node 2 .

Obviously each period 1 quiver $Q$ is also period 2 , where $B(2)=\rho B(1) \rho^{-1}$. However, we will construct some strictly period 2 primitives. 
As before, we have

Lemma 4.2 (Period 2 sink-type equation) Suppose that $Q$ is a quiver with a sink at 1 and that $Q(2)$ has a sink at 2. Then $Q$ is period 2 if and only if $\tau^{2} B_{Q} \tau^{-2}=B_{Q}$.

Proof As before, reversal of the arrows at node 1 of $Q$ can be achieved through a simple conjugation of its matrix: $\mu_{1} B_{Q}=D_{1} B_{Q} D_{1}$. Similarly, reversal of the arrows at node 2 of $Q(2)$ can be achieved through

$$
\mu_{2} B_{Q}(2)=D_{2} B_{Q}(2) D_{2}, \quad \text { where } D_{2}=\operatorname{diag}(1,-1,1, \ldots, 1)=\rho D_{1} \rho^{-1} .
$$

Equating the composition to $\rho^{2} B_{Q} \rho^{-2}$ leads to the equation

$$
B_{Q}=D_{1} D_{2} \rho^{2} B_{Q} \rho^{-2} D_{2} D_{1}=\tau^{2} B_{Q} \tau^{-2} \text {. }
$$

Following the same procedure as for period 1, we need to form orbit-sums for $\tau^{2}$ on the basis considered in the previous section; we shall call these period 2 primitives.

A $\tau$-orbit of odd cardinality is also a $\tau^{2}$-orbit, so the orbit sum will be a period 2 primitive which is also of period 1 . Thus we cannot hope to get period 2 solutions which are not also period 1 solutions unless there are an even number of nodes. A $\tau$-orbit of even cardinality splits into two $\tau^{2}$-orbits.

When $N=2 r$, the matrices $R_{N}^{(k)}$, for $1 \leq k \leq r-1$, generate strictly period 2 primitives $P_{N, 2}^{(k, 1)}$, with matrices given by

$$
B_{N, 2}^{(k, 1)}=\sum_{i=0}^{r-1} \tau^{2 i} R_{N}^{(k)} \tau^{-2 i}
$$

If, in addition, $N$ is divisible by 4 , we obtain the additional strictly period 2 primitives $P_{N, 2}^{(r, 1)}$, with matrices given by

$$
B_{N, 2}^{(r, 1)}=\sum_{i=0}^{r / 2-1} \tau^{2 i} R_{N}^{(r)} \tau^{-2 i}
$$

Geometrically, the primitive $P_{N, 2}^{(k, 1)}$ is obtained from the period 1 primitive $P_{N}^{(k)}$ by "removing half the arrows" (the ones corresponding to odd powers of $\tau$ ). The removed arrows form another period 2 primitive, called $P_{N, 2}^{(k, 2)}$, which may be defined as the matrix:

$$
B_{N, 2}^{(k, 2)}=\tau B_{N, 2}^{(k, 1)} \tau^{-1} .
$$

We make the following observation:

Lemma 4.3 We have

$$
\rho^{-1} \mu_{1} B_{N, 2}^{(k, 1)} \rho=B_{N, 2}^{(k, 2)}
$$

for $1 \leq k \leq r$. 


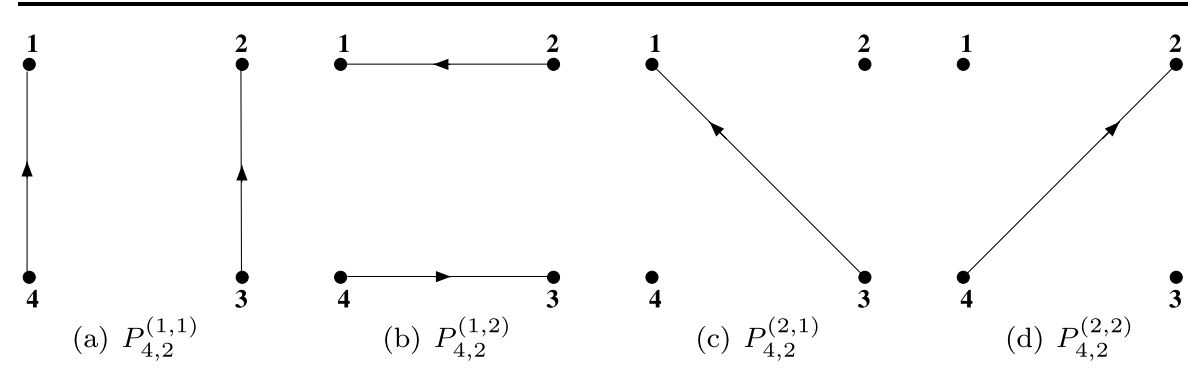

Fig. 5 The strictly period 2 primitives for four nodes

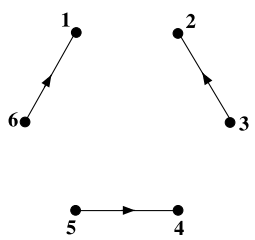

(a) $P_{6,2}^{(1,1)}$

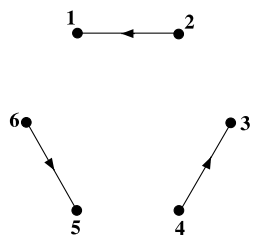

(b) $P_{6,2}^{(1,2)}$

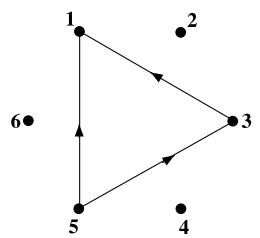

(c) $P_{6,2}^{(2,1)}$

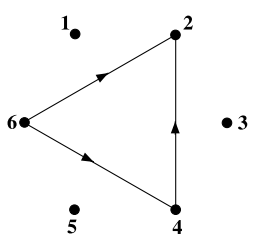

(d) $P_{6,2}^{(2,2)}$

Fig. 6 The period 2 primitives for six nodes

Proof For $1 \leq k \leq r-1$, we have

$$
\begin{aligned}
\rho^{-1} \mu_{1} B_{N, 2}^{(k, 1)} \rho & =\rho^{-1} D_{1} B_{N, 2}^{(k, 1)} D_{1}^{-1} \rho \\
& =\tau^{-1}\left(\sum_{i=0}^{r-1} \tau^{2 i} R_{N}^{(k)} \tau^{-2 i}\right) \tau \\
& =\tau\left(\sum_{i=0}^{r-1} \tau^{2 i-2} R_{N}^{(k)} \tau^{2-2 i}\right) \tau^{-1},
\end{aligned}
$$

since $\rho^{-1} D_{1}=\tau^{-1}$. Since $\tau^{-2}=-\tau^{2 r-2}$, we have $\rho^{-1} B_{N, 2}^{(k, 1)}(2) \rho=\tau B_{N, 1}^{(k, 1)} \tau^{-1}=$ $B_{N, 2}^{(k, 2)}$. A similar argument holds for $k=r$, noting that in this case, $\tau^{-2} R_{N}^{(k)}=$ $\tau^{r-2} R_{N}^{(k)}$.

Figures 5 and 6 show the strictly period 2 primitives with four and six nodes.

We need the following:

\section{Lemma 4.4}

(a) Let $M$ be an $N \times N$ skew-symmetric matrix with $M_{i j} \geq 0$ whenever $i \geq j$. Then $\tau M \tau^{-1}$ has the same property.

(b) All period 2 primitives $B_{N, 2}^{(k, l)}$ have nonnegative entries below the leading diagonal. 
Proof We must also have $M_{i j} \leq 0$ for $i \leq j$. We have

$$
\left(\tau M \tau^{-1}\right)_{i j}= \begin{cases}M_{i-1, j-1} & i>1, j>1, \\ -M_{N, j-1} & i=1, j>1, \\ -M_{i-1, N} & i>1, j=1, \\ M_{N, N} & i=1, j=1\end{cases}
$$

from which (a) follows. Part (b) follows from part (a) and the definition of the period 2 primitives.

As in the period 1 case, we obtain period 2 sink-type quivers by taking orbit-sums of the basis elements:

Proposition 4.5 (Classification of period 2 sink-type quivers) If $N$ is odd, there are no strictly period 2 sink-type quivers with $N$ nodes. If $N=2 r$ is an even integer then every strictly period 2 sink-type quiver with $N$ nodes has corresponding matrix of the form

$$
B= \begin{cases}\sum_{k=1}^{r} \sum_{j=1}^{2} m_{k j} B_{N, 2}^{(k, j)} & \text { if } 4 \mid N, \\ \left(\sum_{k=1}^{r-1} \sum_{j=1}^{2} m_{k j} B_{N, 2}^{(k, j)}\right)+m_{r 1} B_{N}^{(r)} & \text { if } 4 \nmid N,\end{cases}
$$

where the $m_{j k}$ are arbitrary nonnegative integers such that if $4 \mid N$, there is at least one $k, 1 \leq k \leq r$, such that $m_{k 1} \neq m_{k 2}$, and if $4 \nmid N$, there is at least one $k, 1 \leq k \leq$ $r-1$, such that $m_{k 1} \neq m_{k 2}$.

Proof Using the above discussion and an argument similar to that in the period 1 case, we obtain an expression as above for $B$ for which the $m_{k j}$ are integers. It is easy to check that each primitive has a non-zero entry in the first or second column, below the leading diagonal. By Lemma 4.4, this entry must be positive. If the entry is in the first column, the corresponding $m_{k j}$ must be nonnegative as 1 is a sink. If it is in the second column then, since 1 is a sink, mutation at 1 does not affect the entries in the second column below the leading diagonal. Since after mutation at 1,2 is a sink, the corresponding $m_{k j}$ must be nonnegative in this case also.

Whilst the formulae above depend upon particular characteristics of the primitives, i.e. having a specific sink, a similar relation exists for any period 2 quiver. For any quiver $Q$ (regardless of any symmetry or periodicity properties), we have $\mu_{k+1} \rho Q=$ $\rho \mu_{k} Q$, which just corresponds to relabelling the nodes. We write this symbolically as $\mu_{k+1} \rho=\rho \mu_{k}$ and $\rho^{-1} \mu_{k+1}=\mu_{k} \rho^{-1}$. For the period 2 case, the periodic chain (3) can be written as

$$
Q(1) \stackrel{\mu_{1}}{\longrightarrow} Q(2) \stackrel{\mu_{2}}{\longrightarrow} Q(3)=\rho^{2} Q(1) \stackrel{\mu_{3}}{\longrightarrow} Q(4)=\rho^{2} Q(2) \stackrel{\mu_{4}}{\longrightarrow} \cdots .
$$

Whilst $\mu_{1}$ and $\mu_{2}$ are genuinely different mutations, $\mu_{3}$ and $\mu_{4}$ are just $\mu_{1}$ and $\mu_{2}$ after relabelling. Since $\rho^{-1} \mu_{2} Q(2)=\rho Q(1)$, we have $\mu_{1}\left(\rho^{-1} Q(2)\right)=\rho Q(1)$.

We also have $\mu_{2}(\rho Q(1))=\rho \mu_{1} Q(1)=\rho Q(2)$. Since $Q(3)=\mu_{2} Q(2)=$ $\rho^{2} Q(1)$, we have $\rho^{-1} \mu_{2} Q(2)=\rho Q(1)$, and thus we obtain $\mu_{1}\left(\rho^{-1} Q(2)\right)=\rho Q(1)$. We thus can extend the above diagram to that in Fig. 7. 


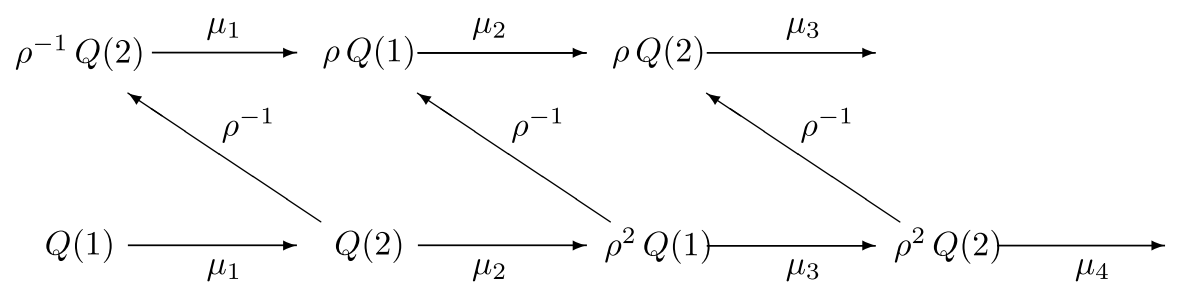

Fig. 7 Period 2 quivers and mutations

If $Q(1), Q(2)$ have sinks at nodes 1 and 2, respectively, then so do $\rho^{-1} Q(2)$ and $\rho Q(1)$ and the mutations $\mu_{1}$ and $\mu_{2}$ in the above diagram act linearly. This gives

$$
\mu_{1}\left(Q(1)+\rho^{-1} Q(2)\right)=Q(2)+\rho Q(1)=\rho\left(Q(1)+\rho^{-1} Q(2)\right)
$$

and

$$
\mu_{2}(Q(2)+\rho Q(1))=\rho^{2} Q(1)+\rho Q(2)=\rho(Q(2)+\rho Q(1)),
$$

so $Q(1)+\rho^{-1} Q(2)$ is period 1 .

We have proved the following:

Proposition 4.6 Let $Q$ be period 2 sink-type quiver. Then $Q(1)+\rho^{-1} Q(2)$ is a quiver of period 1 .

\section{Quivers with higher period}

Higher period primitives are defined in a similar way. The periodic chain (3) contains $m$ quivers $Q(1), Q(2), \ldots, Q(m)$, with corresponding matrices $B(1), \ldots, B(m)$.

Definition 5.1 (Period $m$ sink-type quivers) A quiver $Q$ is said to be a period $m$ sink-type quiver if it is of period $m$ and, for $1 \leq i \leq m$, node $i$ of $Q(i)$ is a sink.

Thus the mutation $Q(i) \mapsto Q(i+1)=\mu_{i} Q(i)$ again only involves the reversal of arrows at node $i$, so can be achieved through a simple conjugation of its matrix: $\mu_{i} B(i)=D_{i} B(i) D_{i}$. Here

$$
D_{i}=\operatorname{diag}(1, \ldots, 1,-1,1, \ldots, 1)=\rho^{i-1} D_{1} \rho^{-i+1}
$$

(with a " -1 " in the $i$ th position).

As in the period 1 and 2 cases, we obtain:

Lemma 5.2 (Period $m$ sink-type equation) Suppose that $Q$ is a quiver with a sink at the $i$ th node of $Q(i)$ for $i=1,2, \ldots, m$. Then $Q$ is period $m$ if and only if $\tau^{m} B_{Q} \tau^{-m}=B_{Q}$. 
Proof We know that $Q$ has period $m$ if and only if $D_{m} \cdots D_{1} B_{Q} D_{1} \cdots D_{m}=$ $\rho^{m} B_{Q} \rho^{-m}$, i.e. if and only if

$$
B_{Q}=D_{1} \cdots D_{m} \rho^{m} B_{Q} \rho^{-m} D_{m} \cdots D_{1}=\tau^{m} B_{Q} \tau^{-m}
$$

Starting with the same matrices $R_{N}^{(k)}$, we now use the action $M \mapsto \tau^{m} M \tau^{-m}$ to build an invariant, i.e. we take orbit sums for $\tau^{m}$. We only obtain strictly $m$-periodic elements in the case where the orbit has size divisible by $m$.

When $m \mid N$, the matrices $R_{N}^{(k)}$, for $1 \leq k \leq r-1$ (where $N=2 r$ or $2 r+1, r$ an integer), generate period $m$ primitives $B_{N, m}^{(k, 1)}$, with matrices given by

$$
B_{N, m}^{(k, 1)}=\sum_{i=0}^{(N / m)-1} \tau^{m i} R_{N}^{(k)} \tau^{-m i}
$$

Geometrically, the primitive $P_{N, m}^{(k, 1)}$ is obtained from the primitive $P_{N}^{(k)}$ by only including every $m$ th arrow. As before, we form another $m-1$ period $m$ primitives, $P_{N, m}^{(k, j)}$ for $j=2, \ldots, m$, with matrices given by

$$
B_{N, m}^{(k, j)}=\tau^{j-1} B_{N, m}^{(k, 1)}\left(\tau^{j-1}\right)^{-1}
$$

Note that the elements $\tau^{l} R_{N}^{(k)} \tau^{-l}$, for $0 \leq l \leq N-1$, form a $\tau$-orbit of size $N$. Since $m \mid N$, this breaks up into $m \tau^{m}$-orbits each of size $N / m$; the elements above are the orbit sums.

Similarly, if $(2 m) \mid N$ (so we are in the case $N=2 r$ ) then the $\tau^{m}$-orbit-sum of $R_{N}^{(r)}$ is

$$
B_{N, m}^{(k, 1)}=\sum_{i=0}^{(N / 2 m)-1} \tau^{m i} R_{N}^{(r)} \tau^{-m i}
$$

with corresponding quiver $P_{N, m}^{(k, 1)}$. We also obtain another $m-1$ period $m$ primitives, $P_{N, m}^{(r, j)}$, for $j=2, \ldots, m$, with matrices

$$
B_{N, m}^{(r, j)}=\tau^{j-1} B_{N, m}^{(r, 1)}\left(\tau^{j-1}\right)^{-1} .
$$

As in the period 1 and 2 cases, we obtain arbitrary strictly period $m$ sink-type quivers by taking orbit-sums of the basis elements. The nonnegativity of the coefficients $m_{k j}$ is shown in a similar way also.

Proposition 5.3 (Classification of period $m$ sink-type quivers) If $m \nmid N$, there are no strictly period $m$ sink-type quivers. If $(2 m) \mid N$, the general strictly period $m$ sink-type quiver is of the form

$$
B=\sum_{k=1}^{r} \sum_{j=1}^{m} m_{k j} B_{N, m}^{(k, j)},
$$


where the $m_{k j}$ are nonnegative integers and there is at least one $k, 1 \leq k \leq r$, for which the $m_{k j}$ are not all equal.

If $m \mid N$ but $(2 m) \nmid N$ then the general period $m$ sink-type quiver has the form

$$
B= \begin{cases}\sum_{k=1}^{r} \sum_{j=1}^{m} m_{k j} B_{N, m}^{(k, j)} & \text { if } N=2 r+1 \text { is odd } \\ \sum_{k=1}^{r-1} \sum_{j=1}^{m} m_{k j} B_{N, m}^{(k, j)}+\sum_{j=1}^{m / 2} m_{r j} B_{N, m / 2}^{(r, j)} & \text { if } N=2 r \text { is even }\end{cases}
$$

where the $m_{k j}$ are nonnegative integers and where in the first case, there is at least one $k, 1 \leq k \leq r$, for which the $m_{k j}$ are not all equal, and in the second case, there is at least one $k, 1 \leq k \leq r-1$, for which the $m_{k j}$ are not all equal.

As before, we use $\mu_{k+1} \rho=\rho \mu_{k}$ and $\rho^{-1} \mu_{k+1}=\mu_{k} \rho^{-1}$, from which it follows that $\mu_{k} \rho^{-j}=\rho^{-j} \mu_{k+j}$. In turn, this gives

$$
\mu_{k}\left(\rho^{-j} Q(j+k)\right)=\rho^{-j} \mu_{j+k} Q(j+k)=\rho^{-j} Q(j+k+1) .
$$

Suppose now that $Q$ is a period $m$ quiver. Then we have $Q(s m+j)=\rho^{s m} Q(j)$ for $1 \leq j \leq m$. We use this to extend the periodic chain (3) to an $m$ level array. We have

$$
\mu_{1}\left(\rho^{-j} Q(j+1)\right)=\rho^{-j} Q(j+2), \quad \mu_{2}\left(\rho^{-j} Q(j+2)\right)=\rho^{-j} Q(j+3), \ldots,
$$

arriving at

$$
\mu_{m}\left(\rho^{-j} Q(j+m)\right)=\rho^{-j} Q(j+m+1)=\rho^{m}\left(\rho^{-j} Q(j+1)\right) .
$$

We write this period $m$ sequence in the $j$ th level of the array, i.e.

$\rho^{-j} Q(j+1) \stackrel{\mu_{1}}{\longrightarrow} \rho^{-j} Q(j+2) \stackrel{\mu_{2}}{\longrightarrow} \cdots \stackrel{\mu_{m-1}}{\longrightarrow} \rho^{-j} Q(j+m) \stackrel{\mu_{m}}{\longrightarrow} \rho^{m}\left(\rho^{-j} Q(j+1)\right)$.

Again we know that if $Q(j)$ has a sink at node $j$ for each $j$, then each $\rho^{-j} Q(j+1)$ has a sink at node 1 and the mutation $\mu_{1}$ acts linearly. This gives

$$
\begin{aligned}
& \mu_{1}\left(Q(1)+\rho^{-1} Q(2)+\cdots+\rho^{-m+1} Q(m)\right) \\
& \quad=\rho\left(Q(1)+\rho^{-1} Q(2)+\cdots+\rho^{-m+1} Q(m)\right),
\end{aligned}
$$

so $Q(1)+\rho^{-1} Q(2)+\cdots+\rho^{-m+1} Q(m)$ is period 1 .

We have proved:

Proposition 5.4 Let $Q$ be period $m$ sink-type quiver. Then $Q(1)+\rho^{-1} Q(2)+\cdots+$ $\rho^{-m+1} Q(m)$ is a quiver of period 1 .

Example 5.5 (Period 3 primitives) Proceeding as described above, whenever $N$ is a multiple of 3 we obtain three period 3 primitives for each period 1 primitive. Figure 8 shows those with six nodes. 


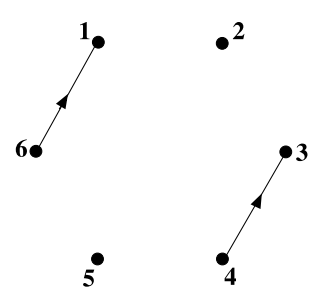

(a) $P_{6,3}^{(1,1)}$

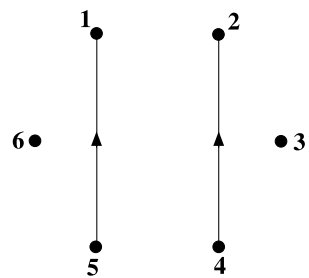

(d) $P_{6,3}^{(2,1)}$

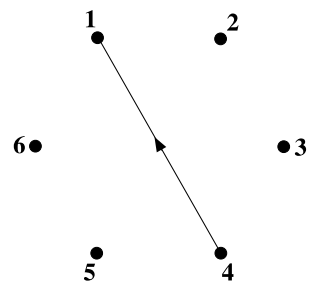

(g) $P_{6,3}^{(3,1)}$

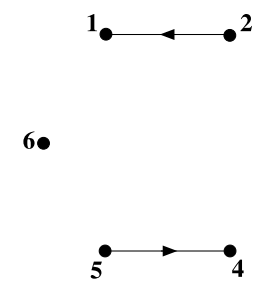

(b) $P_{6,3}^{(1,2)}$

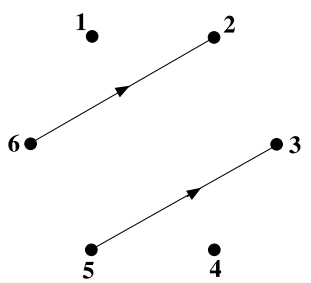

(e) $P_{6,3}^{(2,2)}$

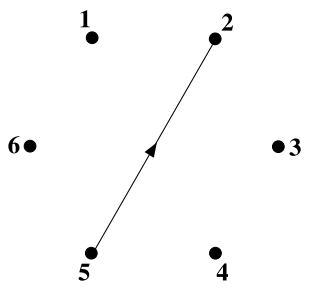

(h) $P_{6,3}^{(3,2)}$

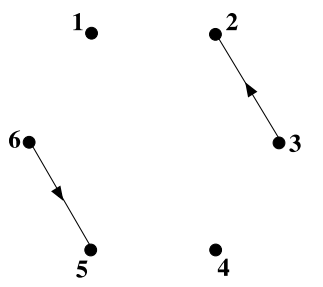

(c) $P_{6,3}^{(1,3)}$

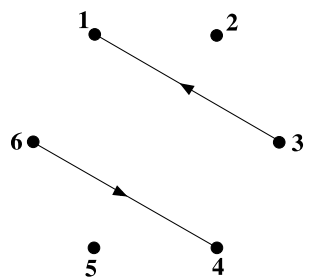

(f) $P_{6,3}^{(2,3)}$

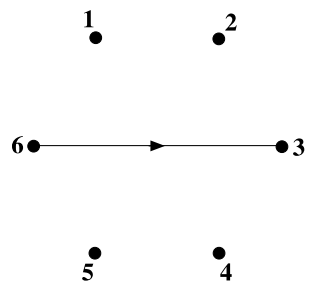

(i) $P_{6,3}^{(3,3)}$

Fig. 8 The period 3 primitives for six nodes

\section{Period 1 general solution}

In this section we give an explicit construction of the $N \times N$ skew-symmetric matrices corresponding to arbitrary period 1 quivers, i.e. those for which mutation at node 1 has the same effect as the rotation $\rho$. We express the general solution as an explicit sum of period 1 primitives, thus giving a simple classification of all such quivers.

In anticipation of the final result, we consider the following matrix:

$$
B=\left(\begin{array}{cccc}
0 & -m_{1} & \cdots & -m_{N-1} \\
m_{1} & 0 & & * \\
\vdots & & 0 & \\
m_{N-1} & * & & 0
\end{array}\right) \text {. }
$$

Using (2), the general mutation rule at node 1 is

$$
\tilde{b}_{i j}= \begin{cases}-b_{i j} & \text { if } i=1 \text { or } j=1, \\ b_{i j}+\frac{1}{2}\left(\left|b_{i 1}\right| b_{1 j}+b_{i 1}\left|b_{1 j}\right|\right) & \text { otherwise. }\end{cases}
$$


The effect of the rotation $B \mapsto \rho B \rho^{-1}$ is to move the entries of $B$ down and right one step, so that $\left(\rho B \rho^{-1}\right)_{i j}=b_{i-1, j-1}$, remembering that indices are labelled modulo $N$, so $N+1 \equiv 1$. For $1 \leq i, j \leq N-1$, let

$$
\varepsilon_{i j}=\frac{1}{2}\left(m_{i}\left|m_{j}\right|-m_{j}\left|m_{i}\right|\right) .
$$

Then if $m_{i}$ and $m_{j}$ have the same sign, $\varepsilon_{i j}=0$. Otherwise $\varepsilon_{i j}= \pm\left|m_{i} m_{j}\right|$, where the sign is that of $m_{i}$. Let $\widetilde{B}=\mu_{1} B$, so that $\tilde{b}_{i j}=b_{i j}+\varepsilon_{i-1, j-1}$.

Theorem 6.1 Let $B$ be an $N \times N$ skew-symmetric integer matrix. Let $b_{k 1}=m_{k-1}$ for $k=2,3, \ldots, N$. Then $\mu_{1} B=\rho B \rho^{-1}$ if and only if $m_{r}=m_{N-r}$ for $r=1,2, \ldots$, $N-1, b_{i j}=m_{i-j}+\varepsilon_{1, i-j+1}+\varepsilon_{2, i-j+2}+\cdots+\varepsilon_{j-1, i-1}$ for all $i>j$, and $B$ is symmetric along the non-leading diagonal.

Proof By skew-symmetry, we note that we only need to determine $b_{i j}$ for $i>j$. We need to solve $\mu_{1} B=\rho B \rho^{-1}$. By the above discussion, this is equivalent to solving

$$
b_{i j}+\varepsilon_{i-1, j-1}=b_{i-1, j-1},
$$

for $i>j$, with $\varepsilon_{i j}$ as given above. Solving the equation leads to a recursive formula for $b_{i j}$.

We obtain

$$
\begin{aligned}
b_{i j} & =b_{i-1, j-1}+\varepsilon_{j-1, i-1} \\
& =b_{i-2, j-2}+\varepsilon_{j-1, i-1}+\varepsilon_{j-2, i-2} \\
& \vdots \\
& =b_{i-j+1,1}+\varepsilon_{j-1, i-1}+\varepsilon_{j-2, i-2}+\cdots+\varepsilon_{1, i-j+1} .
\end{aligned}
$$

In particular, we have

$$
b_{N j}=m_{N-j}+\varepsilon_{1, N-j+1}+\varepsilon_{2, N-j+2}+\cdots+\varepsilon_{j-2, N-2}+\varepsilon_{j-1, N-1} .
$$

We also have $m_{j}=\tilde{b}_{1, j+1}=\left(\rho B \rho^{-1}\right)_{1, j+1}=b_{N j}$. In particular, $m_{1}=b_{N 1}=m_{N-1}$. Equation (8) gives

$$
m_{2}=b_{N 2}=m_{N-2}+\varepsilon_{1, N-1}=m_{N-2}+\varepsilon_{11}=m_{N-2} .
$$

So $m_{2}=m_{N-2}$. Suppose that we have shown that $m_{j}=m_{N-j}$ for $j=1,2, \ldots, r$. Then (8) gives

$$
\begin{aligned}
b_{N, r+1} & =m_{N-r-1}+\varepsilon_{1, N-r}+\varepsilon_{2, N-r+1}+\cdots+\varepsilon_{r, N-1} \\
& =m_{N-r-1}+\sum_{i=1}^{r} \varepsilon_{i, N-r+i-1}
\end{aligned}
$$




$$
\begin{aligned}
& =m_{N-r-1}+\sum_{i=1}^{r} \varepsilon_{i, r+1-i} \\
& =m_{N-r-1}+\varepsilon_{1, r}+\varepsilon_{2, r-1}+\cdots+\varepsilon_{r, 1}=m_{N-r-1},
\end{aligned}
$$

using the inductive hypothesis and the fact that $\varepsilon_{s t}=-\varepsilon_{t s}$ for all $s, t$. Hence $m_{r+1}=$ $m_{N-r-1}$ and we have by induction that $m_{r}=m_{N-r}$ for $1 \leq r \leq N-1$.

We have, for $i>j$, by (8),

$$
\begin{aligned}
b_{N-j+1, N-i+1}= & m_{(N-j+1)-(N-i+1)}+\varepsilon_{(N-i+1)-1,(N-j+1)-1} \\
& +\varepsilon_{(N-i+1)-2,(N-j+1)-2}+\cdots+\varepsilon_{1,(N-j+1)-(N-i+1)+1} \\
= & m_{i-j}+\varepsilon_{N-i, N-j}+\varepsilon_{N-i-1, N-j-1}+\cdots+\varepsilon_{1, i-j+1},
\end{aligned}
$$

and we have, again using (8) and the fact that $\varepsilon_{N-a, N-b}=\varepsilon_{a b}$,

$$
\begin{aligned}
m_{i-j}=m_{N-i+j}= & b_{N, N-i+j} \\
= & b_{N-j+1, N-i+1}+\varepsilon_{N-i+j-1, N-1}+\varepsilon_{N-i+j-2, N-2}+\cdots \\
& \quad+\varepsilon_{N-i+1, N-j+1} \\
= & b_{N-j+1, N-i+1}+\varepsilon_{i-j+1,1}+\varepsilon_{i-j+2,2}+\cdots+\varepsilon_{i-1, j-1},
\end{aligned}
$$

so

$$
b_{N-j+1, N-i+1}=m_{i-j}+\varepsilon_{j-1, i-1}+\cdots+\varepsilon_{i-j+2,2}+\varepsilon_{1, i-j+1}=b_{i j} .
$$

Hence $B$ is symmetric along the non-leading diagonal.

If $B$ satisfies all the requirements in the statement of the theorem, then (8) is satisfied, and therefore $\rho B \rho^{-1}=\mu_{1} B$. The proof is complete.

We remark that with the identification $m_{r}=m_{N-r}$, we have seen that the formula (8) has a symmetry, due to which the $\varepsilon$ 's cancel in pairwise fashion:

$$
b_{N, N-k+1}=b_{k 1}+\varepsilon_{1 k}+\varepsilon_{2, k+1}+\cdots+\varepsilon_{k+1,2}+\varepsilon_{k 1} .
$$

The formula (8) is just a truncation of this, so not all terms cancel. As we march from $b_{k 1}$ in a "south easterly direction", we first add $\varepsilon_{1 k}, \varepsilon_{2, k+1}$, etc., until we reach $\varepsilon_{r, r+1}$ (when $N-k=2 r$ ) or $\varepsilon_{r r}=0$ (when $N-k=2 r+1$ ). At this stage we start to subtract terms on a basis of "last in, first out", with the result that the matrix has reflective symmetry about the second diagonal as we have seen.

Remark 6.2 (Sink-type case) We note that if all the $m_{i}$ have the same sign, then all the $\varepsilon_{i j}$ are zero. Equation (7) reduces to $b_{i j}=b_{i-1, j-1}$ and we recover the sink-type period 1 solutions considered in Proposition 3.6.

\subsection{Examples}

The simplest nontrivial example is when $N=4$. 
Example 6.3 (Period 1 quiver with four nodes) Here the matrix has the form

$$
B=\left(\begin{array}{cccc}
0 & -m_{1} & -m_{2} & -m_{1} \\
m_{1} & 0 & -m_{1}-\varepsilon_{12} & -m_{2} \\
m_{2} & m_{1}+\varepsilon_{12} & 0 & -m_{1} \\
m_{1} & m_{2} & m_{1} & 0
\end{array}\right) .
$$

As previously noted, if $m_{1}$ and $m_{2}$ have the same sign, then $\varepsilon_{12}=0$ and this matrix is just the sum of primitives for four nodes. The $2 \times 2$ matrix in the "centre" of $B$ (formed out of rows and columns 2 and 3),

$$
\left(\begin{array}{cc}
0 & -\varepsilon_{12} \\
\varepsilon_{12} & 0
\end{array}\right),
$$

corresponds to $\varepsilon_{12}$ times the primitive $P_{2}^{(1)}$ with two nodes (see Fig. 2). For the case $m_{1}=1, m_{2}=-2, \varepsilon_{12}=2$, we obtain the Somos 4 quiver in Fig. 1(a). The action of $\iota$ (see Remark 3.5 ) is $1 \leftrightarrow 4,2 \leftrightarrow 3$ and clearly just reverses all the arrows as predicted by Remark 3.5 .

Example 6.4 (Period 1 quiver with five nodes) Here the general period 1 solution has the form

$$
B=\left(\begin{array}{ccccc}
0 & -m_{1} & -m_{2} & -m_{2} & -m_{1} \\
m_{1} & 0 & -m_{1}-\varepsilon_{12} & -m_{2}-\varepsilon_{12} & -m_{2} \\
m_{2} & m_{1}+\varepsilon_{12} & 0 & -m_{1}-\varepsilon_{12} & -m_{2} \\
m_{2} & m_{2}+\varepsilon_{12} & m_{1}+\varepsilon_{12} & 0 & -m_{1} \\
m_{1} & m_{2} & m_{2} & m_{1} & 0
\end{array}\right)
$$

which can be written as

$$
B=\sum_{k=1}^{2} m_{k} B_{5}^{(k)}+\varepsilon_{12} B_{3}^{(1)},
$$

where $B_{3}^{(1)}$ is embedded symmetrically in the middle of a $5 \times 5$ matrix (surrounded by zeros).

When $m_{1}=1$ and $m_{2}=-1$, this matrix corresponds to the Somos 5 sequence; see Fig. 9 for the corresponding quiver.

Fig. 9 The Somos 5 quiver

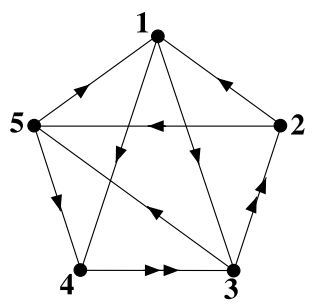


Example 6.5 (Period 1 quiver with six nodes) Here the matrix has the form

$$
\begin{aligned}
B= & \left(\begin{array}{cccccc}
0 & -m_{1} & -m_{2} & -m_{3} & -m_{2} & -m_{1} \\
m_{1} & 0 & -m_{1} & -m_{2} & -m_{3} & -m_{2} \\
m_{2} & m_{1} & 0 & -m_{1} & -m_{2} & -m_{3} \\
m_{3} & m_{2} & m_{1} & 0 & -m_{1} & -m_{2} \\
m_{2} & m_{3} & m_{2} & m_{1} & 0 & -m_{1} \\
m_{1} & m_{2} & m_{3} & m_{2} & m_{1} & 0
\end{array}\right) \\
& +\left(\begin{array}{c|cccc|c}
0 & 0 & 0 & 0 & 0 & 0 \\
\hline 0 & 0 & -\varepsilon_{12} & -\varepsilon_{13} & -\varepsilon_{12} & 0 \\
0 & \varepsilon_{12} & 0 & -\varepsilon_{12} & -\varepsilon_{13} & 0 \\
0 & \varepsilon_{13} & \varepsilon_{12} & 0 & -\varepsilon_{12} & 0 \\
0 & \varepsilon_{12} & \varepsilon_{13} & \varepsilon_{12} & 0 & 0 \\
\hline 0 & 0 & 0 & 0 & 0 & 0
\end{array}\right)+\left(\begin{array}{cc|cc|cc}
0 & 0 & 0 & 0 & 0 & 0 \\
0 & 0 & 0 & 0 & 0 & 0 \\
\hline 0 & 0 & 0 & -\varepsilon_{23} & 0 & 0 \\
0 & 0 & \varepsilon_{23} & 0 & 0 & 0 \\
\hline 0 & 0 & 0 & 0 & 0 & 0 \\
0 & 0 & 0 & 0 & 0 & 0
\end{array}\right),
\end{aligned}
$$

which can be written as

$$
B=\sum_{j=1}^{3} m_{k} B_{6}^{(k)}+\sum_{k=1}^{2} \varepsilon_{1, k+1} B_{4}^{(k)}+\varepsilon_{23} B_{2}^{(1)},
$$

where the periodic solutions with fewer rows and columns are embedded symmetrically within a $6 \times 6$ matrix.

\subsection{The period 1 general solution in terms of primitives}

It can be seen from the above examples that the solutions are built out of a sequence of sub-matrices, each of which corresponds to one of the primitives. The main matrix is just an integer linear combination of primitive matrices for the full set of $N$ nodes. The next matrix is a combination (with coefficients $\varepsilon_{1 j}$ ) of primitive matrices for the $N-2$ nodes $2, \ldots, N-1$. We continue to reduce by 2 until we reach either two nodes (when $N$ is even) or three nodes (when $N$ is odd).

Remarkably, as can be seen from the general structure of the matrix given by (8), together with the symmetry $m_{N-r}=m_{r}$, this description holds for all $N$.

Recall that for an even (or odd) number of nodes, $N=2 r$ (or $N=2 r+1$ ), there are $r$ primitives, labelled $B_{2 r}^{(k)}$ (or $\left.B_{2 r+1}^{(k)}\right), k=1, \ldots, r$. We denote the general linear combination of these by

$$
\widetilde{B}_{2 r}\left(\mu_{1}, \ldots, \mu_{r}\right)=\sum_{j=1}^{r} \mu_{j} B_{2 r}^{(j)}, \quad \text { or } \quad \widetilde{B}_{2 r+1}\left(\mu_{1}, \ldots, \mu_{r}\right)=\sum_{j=1}^{r} \mu_{j} B_{2 r+1}^{(j)} \text {, }
$$

for integers $\mu_{j}$.

The quivers corresponding to $\widetilde{B}_{2 r}\left(\mu_{1}, \ldots, \mu_{r}\right)$ and $\widetilde{B}_{2 r+1}\left(\mu_{1}, \ldots, \mu_{r}\right)$ (i.e. without the extra terms coming from the $\varepsilon_{i j}$ ) do not have periodicity properties (in general).

We now restate Theorem 6.1 in this new notation: 
Theorem 6.6 (The general period 1 quiver) Let $B_{2 r}$ (respectively $B_{2 r+1}$ ) denote the matrix corresponding to the general even (respectively odd) node quiver of mutation periodicity 1 . Then

1.

$$
B_{2 r}=\widetilde{B}_{2 r}\left(m_{1}, \ldots, m_{r}\right)+\sum_{k=1}^{r-1} \widetilde{B}_{2(r-k)}\left(\varepsilon_{k, k+1}, \ldots, \varepsilon_{k r}\right),
$$

where the matrix $\widetilde{B}_{2(r-k)}\left(\varepsilon_{k, k+1}, \ldots, \varepsilon_{k r}\right)$ is embedded in a $2 r \times 2 r$ matrix in rows 2 . and columns $k+1, \ldots, 2 r-k$.

$$
B_{2 r+1}=\widetilde{B}_{2 r+1}\left(m_{1}, \ldots, m_{r}\right)+\sum_{k=1}^{r-1} \widetilde{B}_{2(r-k)+1}\left(\varepsilon_{k, k+1}, \ldots, \varepsilon_{k r}\right)
$$

where the matrix $\widetilde{B}_{2(r-k)+1}\left(\varepsilon_{k, k+1}, \ldots, \varepsilon_{k r}\right)$ is embedded in $a(2 r+1) \times(2 r+1)$ matrix in rows and columns $k+1, \ldots, 2 r+1-k$.

\section{Quivers with mutation periodicity 2}

Already at period 2, we cannot give a full classification of the possible quivers. However, we can give the full list for low values of $N$, the number of nodes. We can also give a class of period 2 quivers which exists for odd or even $N$.

When $N$ is even, primitives play a role, but the full solution cannot be written purely in terms of primitives. When $N$ is $o d d$, primitives do not even exist, but there are still quivers with mutation periodicity 2 .

Consider the period 2 chain:

$$
Q(1) \stackrel{\mu_{1}}{\longrightarrow} Q(2) \stackrel{\mu_{2}}{\longrightarrow} Q(3)=\rho^{2} Q(1) .
$$

A simpler way to compute is to use $\mu_{2} Q(3)=Q(2)$, so $\mu_{2} \rho^{2} Q(1)=Q(2)$. Hence we must solve

$$
\rho \mu_{1} \rho Q(1)=\mu_{1} Q(1)
$$

which are the equations referred to below. We first consider the solution of these equations for $N=3, \ldots, 5$.

We need one new piece of notation, which generalises our former $\varepsilon_{i j}$. We define

$$
\varepsilon(x, y)=\frac{1}{2}(x|y|-y|x|) .
$$

Thus, $\varepsilon_{i j}=\varepsilon\left(m_{i}, m_{j}\right)$. 


\subsection{3 node quivers of period 2}

Let

$$
B(1)=\left(\begin{array}{ccc}
0 & -m_{1} & -m_{2} \\
m_{1} & 0 & -b_{32} \\
m_{2} & b_{32} & 0
\end{array}\right) .
$$

Equation (9) gives the equalities $m_{1}=b_{32}$ and $m_{2}-m_{1}=-\varepsilon_{12}$. If the signs of $m_{1}$ and $m_{2}$ are the same, we obtain a period 1 solution. Assuming otherwise leads to the equation $m_{2}-m_{1}= \pm m_{1} m_{2}$ depending on the sign of $m_{1}$ (and $m_{2}$ ). The only integer solutions to this equation are $m_{1}= \pm 2$ and $m_{2}=\mp 2$.

It follows that there are just two solutions of period two: the following matrix and its negative:

$$
B(1)=\left(\begin{array}{ccc}
0 & -2 & 2 \\
2 & 0 & -2 \\
-2 & 2 & 0
\end{array}\right) .
$$

This corresponds to a 3-cycle of double arrows. Notice that in this case, there are no free parameters. Mutating at node 1 just gives $B(2)=-B(1)$, i.e. $Q(2)=Q(1)^{o p p}$. Note that the representation theoretic properties of this quiver are discussed at some length in $[4, \S 8, \S 11]$.

\subsection{4 node quivers of period 2}

We start with the matrix

$$
B(1)=\left(\begin{array}{cccc}
0 & -m_{1} & -m_{2} & -m_{3} \\
m_{1} & 0 & -b_{32} & b_{42} \\
m_{2} & b_{32} & 0 & -b_{43} \\
m_{3} & b_{42} & b_{43} & 0
\end{array}\right) .
$$

Setting $p_{1}=b_{42}$ and solving for $b_{43}$ and $b_{32}$ in terms of $p_{1}$ and the $m_{i}$ 's we find

$$
b_{43}=m_{1}, \quad \text { and } \quad b_{32}=m_{3}+\varepsilon\left(m_{1}, p_{1}\right) .
$$

We also obtain the three conditions

$$
\varepsilon_{13}=0, \quad \varepsilon_{12}-\varepsilon\left(m_{1}, p_{1}\right)=0, \quad \varepsilon_{23}+\varepsilon\left(m_{3}, p_{1}\right)=0 .
$$

The first of these three conditions just means that $m_{1}$ and $m_{3}$ have the same sign (or that one of them is zero). Choosing $m_{1}>0$, so $m_{3} \geq 0$, we must have $m_{2}<0$ for node 1 not to be a sink. The remaining conditions are then

$$
m_{1}\left(\left|p_{1}\right|-p_{1}+2 m_{2}\right)=0, \quad m_{3}\left(\left|p_{1}\right|-p_{1}+2 m_{2}\right)=0 .
$$


For a nontrivial solution we must have $p_{1}<0$, which leads to $p_{1}=m_{2}$. The final result is then the following:

$$
\begin{aligned}
& B(1)=\left(\begin{array}{cccc}
0 & -m_{1} & -m_{2} & -m_{3} \\
m_{1} & 0 & m_{1} m_{2}-m_{3} & -m_{2} \\
m_{2} & m_{3}-m_{1} m_{2} & 0 & -m_{1} \\
m_{3} & m_{2} & m_{1} & 0
\end{array}\right), \\
& B(2)=\left(\begin{array}{cccc}
0 & m_{1} & m_{2} & m_{3} \\
-m_{1} & 0 & -m_{3} & -m_{2} \\
-m_{2} & m_{3} & 0 & m_{2} m_{3}-m_{1} \\
-m_{3} & m_{2} & m_{1}-m_{2} m_{3} & 0
\end{array}\right),
\end{aligned}
$$

with $m_{1}>0, m_{2}<0$ and $m_{3} \geq 0$. Notice that $B(2)\left(m_{1}, m_{3}\right)=\rho B(1)\left(m_{3}, m_{1}\right) \rho^{-1}$, so the period 2 property stems from the involution $m_{1} \leftrightarrow m_{3}$. If $m_{3}=m_{1}$, then the quiver has mutation period 1. We may choose either of these to be zero, but not $m_{2}$, since, again, node 1 would be a sink.

Remark 7.1 (The quiver and its opposite) We made the choice that $m_{1}>0$. The equivalent choice $m_{1}<0$ would just lead to the negative of $B(1)$, corresponding to $Q(1)^{o p p}$.

Remark 7.2 (A graph symmetry) Notice that all 4-node quivers of period 2 have the graph symmetry $(1,2,3,4) \leftrightarrow(4,3,2,1)$, under which $Q \mapsto Q^{o p p}$.

For $N \geq 5$, we cannot construct the general solution of (9) without further assumptions. However, we can find some solutions and these also have this graph symmetry. Furthermore, if we assume the graph symmetry, then we can find the general solution for some higher values of $N$, but have no general proof that this will be the case for all $N$.

We previously saw this graph symmetry in the context of period 1 primitives (see Remark 3.5).

\subsection{5 node quivers of period 2}

Starting with the general skew-symmetric, $5 \times 5$ matrix, with

$$
b_{k 1}=m_{k-1}, \quad k=2, \ldots, 5 \text { and } b_{52}=p_{1},
$$

we immediately find

$$
\begin{aligned}
& b_{32}=m_{4}+\varepsilon_{12}, \quad b_{42}=m_{2}+\varepsilon_{14}+\varepsilon\left(m_{1}, p_{1}\right), \\
& b_{43}=m_{4}+\varepsilon\left(m_{1}, p_{1}-\varepsilon_{14}\right), \quad b_{53}=p_{1}-\varepsilon_{14}, \quad b_{54}=m_{1},
\end{aligned}
$$

together with the simple condition $m_{3}=m_{2}+\varepsilon_{14}$ and four complicated conditions.

Imposing the graph symmetry (Remark 7.2) leads to $p_{1}=m_{2}=\varepsilon_{14}$, after which two of the four conditions are identically satisfied, whilst the other pair reduce to a single condition:

$$
\varepsilon\left(m_{2}, p_{1}\right)+\varepsilon\left(m_{4}, p_{1}\right)-\varepsilon 12=m_{4}-m_{1} .
$$


We need integer solutions for $m_{1}, m_{2}, m_{4}$. There are a number of subcases.

The case $m_{1}>0, m_{4}>0$

In this case the remaining condition reduces to

$$
\left(\left|m_{2}\right|-m_{2}-2\right)\left(m_{1}-m_{4}\right)=0 .
$$

Discarding the period 1 solution, $m_{4}=m_{1}$, we obtain $m_{2}=-1$, leading to

$$
\begin{gathered}
B(1)=\left(\begin{array}{ccccc}
0 & -m_{1} & 1 & 1 & -m_{4} \\
m_{1} & 0 & -m_{1}-m_{4} & 1-m_{1} & 1 \\
-1 & m_{1}+m_{4} & 0 & -m_{1}-m_{4} & 1 \\
-1 & m_{1}-1 & m_{1}+m_{4} & 0 & -m_{1} \\
m_{4} & -1 & -1 & m_{1} & 0
\end{array}\right), \\
B(2)=\left(\begin{array}{ccccc}
0 & m_{1} & -1 & -1 & m_{4} \\
-m_{1} & 0 & -m_{4} & 1 & 1 \\
1 & m_{4} & 0 & -m_{1}-m_{4} & 1-m_{4} \\
1 & -1 & m_{1}+m_{4} & 0 & -m_{1}-m_{4} \\
-m_{4} & -1 & m_{4}-1 & m_{1}+m_{4} & 0
\end{array}\right) .
\end{gathered}
$$

Notice again that $B(2)\left(m_{1}, m_{4}\right)=\rho B(1)\left(m_{4}, m_{1}\right) \rho^{-1}$, so the period 2 property stems from the involution $m_{1} \leftrightarrow m_{4}$.

The case $m_{1}>0, m_{4}<0, m_{2}>0$

There is one condition, which can be reduced by noting that $m_{2}-m_{1} m_{4}>0$, giving

$$
m_{4}\left(m_{2}-1\right)=m_{1}\left(m_{4}^{2}-1\right) .
$$

The left side is negative and the right positive unless $m_{2}=1, m_{4}=-1$. We then have $m_{3}=p_{1}=m_{1}+1$, giving

$$
\begin{gathered}
B(1)=\left(\begin{array}{ccccc}
0 & -m_{1} & -1 & -m_{1}-1 & 1 \\
m_{1} & 0 & 1 & -m_{1}-1 & -m_{1}-1 \\
1 & -1 & 0 & 1 & -1 \\
m_{1}+1 & m_{1}+1 & -1 & 0 & -m_{1} \\
-1 & m_{1}+1 & 1 & m_{1} & 0
\end{array}\right), \\
B(2)=\left(\begin{array}{ccccc}
0 & m_{1} & 1 & m_{1}+1 & -1 \\
-m_{1} & 0 & 1 & -m_{1}-1 & -1 \\
-1 & -1 & 0 & 1 & 0 \\
-m_{1}-1 & m_{1}+1 & -1 & 0 & 1 \\
1 & 1 & 0 & -1 & 0
\end{array}\right) .
\end{gathered}
$$


The case $m_{1}>0, m_{4}<0, m_{2}<0$

Here we have no control over the sign of $m_{2}-m_{1} m_{4}$.

When $m_{2}-m_{1} m_{4}>0$, we have the single condition

$$
\left(m_{2}-m_{1} m_{4}\right)\left(m_{2}+m_{4}\right)+m_{1}\left(m_{2}+1\right)-m_{4}=0 .
$$

Whilst any integer solution would give an example, we have no way of determining these. (However, Andy Hone has communicated to us that an algebraic-numbertheoretic argument can be used to show that there are no integer solutions.)

When $m_{2}-m_{1} m_{4}<0$, we have $m_{4}=m_{1}\left(m_{2}+1\right)$, so $m_{3}=m_{2}-m_{1} m_{4}=$ $m_{2}-m_{1}^{2}\left(m_{2}+1\right)$. We must have $m_{2} \leq-2$ for $m_{4}<0$. Since $m_{2}-m_{1} m_{4}=m_{2}-$ $m_{1}^{2}\left(m_{2}+1\right)<0$, we then choose $m_{1}$ to be any integer satisfying $m_{1}>\sqrt{\frac{m_{2}}{m_{2}+1}}$.

Subject to these constraints, the matrices take the form:

$$
\begin{array}{r}
B(1)=\left(\begin{array}{ccccc}
0 & -m_{1} & -m_{2} & -m_{3} & -m_{1}\left(m_{2}+1\right) \\
m_{1} & 0 & -m_{1} & m_{3}\left(m_{1}-1\right) & -m_{3} \\
m_{2} & m_{1} & 0 & -m_{1} & -m_{2} \\
m_{3} & -m_{3}\left(m_{1}-1\right) & m_{1} & 0 & -m_{1} \\
m_{1}\left(m_{2}+1\right) & m_{3} & m_{2} & m_{1} & 0
\end{array}\right), \\
B(2)=\left(\begin{array}{ccccc}
0 & m_{1} & m_{2} & m_{3} & m_{1}\left(m_{2}+1\right) \\
-m_{1} & 0 & -m_{1}\left(m_{2}+1\right) & m_{3} & -m_{2} \\
-m_{2} & m_{1}\left(m_{2}+1\right) & 0 & -m_{1} & -m_{2} \\
-m_{3} & -m_{3} & m_{1} & 0 & -m_{1} \\
-m_{1}\left(m_{2}+1\right) & m_{2} & m_{2} & m_{1} & 0
\end{array}\right) .
\end{array}
$$

The simplest solution has $m_{1}=2, m_{2}=-2$.

\subsection{A family of period 2 solutions}

We are not able to classify all period 2 quivers. Note that in Sect. 4 we have classified all sink-type period 2 quivers. In this section we shall explain how to modify the proof of the classification of period 1 quivers (see Sect. 6) in order to construct a family of period 2 quivers (which are, in general, not sink-type). The introduction of the involution $\sigma$, defined below, is motivated by the matrices (10) and (11).

As before, we consider the matrix:

$$
B=\left(\begin{array}{cccc}
0 & -m_{1} & \cdots & -m_{N-1} \\
m_{1} & 0 & & * \\
\vdots & & 0 & \\
m_{N-1} & * & & 0
\end{array}\right) .
$$

However, we assume that, for $r=2,3, \ldots, N-2, m_{r}=m_{N-r}$ (in the period 1 case this property follows automatically). We write $m_{1}$ instead of $m_{N-1}$, for convenience. 
We also assume that $m_{1} \geq 0, m_{N-1}=m_{\overline{1}} \geq 0$ and $m_{1} \neq m_{\overline{1}}$ (the last condition to ensure we obtain strictly period 2 matrices). We consider the involution $\sigma$ which fixes $m_{r}$ for $r \neq 1$ and interchanges $m_{1}$ and $m_{\overline{1}}$. Let $\mathbf{m}=\left(m_{1}, m_{2}, \ldots, m_{N-2}, m_{\overline{1}}\right)$. We write $\sigma(\mathbf{m})=\sigma\left(m_{1}, m_{2}, \ldots, m_{N-2}, m_{\overline{1}}\right)=\left(m_{\overline{1}}, m_{2}, \ldots, m_{N-2}, m_{1}\right)$.

Our aim is to construct a matrix $B=B\left(m_{1}, m_{2}, \ldots, m_{N-1}\right)$ which satisfies the equation

$$
\mu_{1}(B)=\rho B(\sigma(\mathbf{m})) \rho^{-1} .
$$

Since $\sigma$ is an involution, we shall obtain period 2 solutions in this way. As in the period 1 case, (15) implies that $\left(b_{N 1}, b_{N 2}, \ldots, b_{N, N-1}\right)=\sigma(\mathbf{m})$. The derivation of (7) in the period 1 case is modified by the action of $\sigma$ to give

$$
b_{i j}=\sigma\left(b_{i-1, j-1}\right)+\varepsilon_{j-1, i-1} .
$$

An easy induction shows that

$$
b_{i j}=\sigma^{j-1}\left(b_{i-j+1,1}\right)+\sum_{s=1}^{j-1} \sigma^{j-1-s}\left(\varepsilon_{s, i-j+s}\right) .
$$

Applying this in the case $i=N$ we obtain

$$
b_{N j}=\sigma^{j-1}\left(b_{N-j+1,1}\right)+\sum_{s=1}^{j-1} \sigma^{j-1-s}\left(\varepsilon_{s, N-j+s}\right) .
$$

Hence we have

$$
b_{N j}=\sigma^{j-1}\left(b_{N-j+1,1}\right)+\varepsilon_{j-1, \overline{1}}+\sum_{s=1}^{j-2} \sigma^{j-1-s}\left(\varepsilon_{s, j-s)}\right) .
$$

For $j \leq N-2$, this gives

$$
m_{j}=m_{N-j}+\sigma^{j-2}\left(\varepsilon_{1, j-1}\right)+\varepsilon_{j-1, \overline{1}} .
$$

Since $m_{j}=m_{N-j}$, this is equivalent to $\sigma^{j-2}\left(\varepsilon_{1, j-1}\right)+\varepsilon_{j-1, \overline{1}}=0$. For $j=2$ this is automatically satisfied, since $\varepsilon_{1 \overline{1}}=0$. For $j \geq 3$ and odd, this is always true. For $j \geq 4$ and even, this is true if and only if $m_{j-1} \geq 0$. For $j=N-1$, we obtain

$$
m_{1}=b_{N, N-1}=\sigma^{N-2}\left(m_{1}\right)+\varepsilon_{2,1}+\sum_{s=1}^{N-3} \sigma^{N-2-s}\left(\varepsilon_{s, j-s}\right)
$$

and thus

$$
m_{1}=\sigma^{N-2}\left(m_{1}\right)+\sigma^{N-3}\left(\varepsilon_{1,2}\right)+\varepsilon_{2, \overline{1}} .
$$

For $N$ even this is equivalent to $\varepsilon_{1,2}+\varepsilon_{2, \overline{1}}=0$, which always holds. For $N$ odd this gives the condition

$$
m_{1}=m_{\overline{1}}+\varepsilon_{1,2}+\varepsilon_{2, \overline{1}} .
$$


If $m_{2} \geq 0$, this is equivalent to $m_{1}=m_{1}$, a contradiction to our assumption. If $m_{2}<0$, this is equivalent to $m_{1}=m_{\overline{1}}-m_{1} m_{2}+m_{2} m_{\overline{1}}$, which holds if and only if $m_{2}=-1$ (since we have assumed that $m_{1} \neq m_{-}$). Therefore, we obtain a period 2 solution provided $m_{r} \geq 0$ for $r$ odd, $r \geq 3$ and, in addition, $m_{2}=-1$ for $N$ odd.

\section{Recurrences with the Laurent property}

As previously said, our original motivation for this work was the well known connection between cluster algebras and sequences with the Laurent property, developed by Fomin and Zelevinsky in [7, 8]. We note that cluster algebras were initially introduced (in [7]) in order to study total positivity of matrices and the (dual of the) canonical basis of Kashiwara [20] and Lusztig [23] for a quantised enveloping algebra.

In this section we use the cluster algebras associated to periodic quivers to construct sequences with the Laurent property. These are likely to be a rich source of integrable maps. Indeed, it is well known (see [19]) that the Somos 4 recurrence can be viewed as an integrable map, having a degenerate Poisson bracket and first integral, which can be reduced to a 2-dimensional symplectic map with first integral. This 2-dimensional map is a special case of the QRT [27] family of integrable maps. The Somos 4 Poisson bracket is a special case of that introduced in [14] for all cluster algebra structures. For many of the maps derived by the construction given in this section, it is also possible to construct first integrals, often enough to prove complete integrability. We do not yet have a complete picture, so do not discuss this property in general. However, the maps associated with our primitives are simple enough to treat in general and can even be linearised. This is presented in Sect. 9.

A (skew-symmetric, coefficient-free) cluster algebra is an algebraic structure which can be associated with a quiver. (Recall that we only consider quivers with no 1- or 2-cycles.) Given a quiver (with $N$ nodes), we attach a variable at each node, labelled $\left(x_{1}, \ldots, x_{N}\right)$. When we mutate the quiver we change the associated matrix according to formula (2) and, in addition, we transform the cluster variables $\left(x_{1}, \ldots, x_{N}\right) \mapsto\left(x_{1}, \ldots, \tilde{x}_{\ell}, \ldots, x_{N}\right)$, where

$$
x_{\ell} \tilde{x}_{\ell}=\prod_{b_{i \ell}>0} x_{i}^{b_{i \ell}}+\prod_{b_{i \ell}<0} x_{i}^{-b_{i \ell}}, \quad \tilde{x}_{i}=x_{i} \text { for } i \neq \ell .
$$

If one of these products is empty (which occurs when all $b_{i \ell}$ have the same sign) then it is replaced by the number 1 . This formula is called the (cluster) exchange relation. Notice that it just depends upon the $\ell$ th column of the matrix. Since the matrix is skew-symmetric, the variable $x_{\ell}$ does not occur on the right side of (17).

After this process we have a new quiver $\tilde{Q}$, with a new matrix $\tilde{B}$. This new quiver has cluster variables $\left(\tilde{x}_{1}, \ldots, \tilde{x}_{N}\right)$. However, since the exchange relation (17) acts as the identity on all except one variable, we write these new cluster variables as $\left(x_{1}, \ldots, \tilde{x}_{\ell}, \ldots, x_{N}\right)$. We can now repeat this process and mutate $\tilde{Q}$ at node $p$ and produce a third quiver $\tilde{\tilde{Q}}$, with cluster variables $\left(x_{1}, \ldots, \tilde{x}_{\ell}, \ldots, \tilde{x}_{p}, \ldots, x_{N}\right)$, with $\tilde{x}_{p}$ being given by an analogous formula (17), but using variable $\tilde{x}_{\ell}$ instead of $x_{\ell}$. 
Remark 8.1 (Involutive property of the exchange relation) Since the matrix mutation formula (2) just changes the signs of the entries in column $n$, a second mutation at this node would entail an identical right hand side of (17) (just interchanging the two products), leading to

$$
\tilde{x}_{\ell} \tilde{\tilde{x}}_{\ell}=x_{\ell} \tilde{x}_{\ell} \quad \Rightarrow \quad \tilde{\tilde{x}}_{\ell}=x_{\ell}
$$

Therefore, the exchange relation is an involution.

Remark 8.2 (Equivalence of a quiver and its opposite) The mutation formula (17) for a quiver and its opposite are identical since this corresponds to just a change of sign of the matrix entries $b_{i \ell}$. This is a reason for considering these quivers as equivalent in our context.

In this paper we have introduced the notion of mutation periodicity and followed the convention that we mutate first at node 1, then at node 2, etc. Mutation periodicity (period $m$ ) meant that after $m$ steps we return to a quiver which is equivalent (up to a specific permutation) to the original quiver $Q$ (see the diagram (3)). The significance of this is that the mutation at node $m+1$ produces an exchange relation which is identical in form (but with a different labelling) to the exchange relation at node 1 . The next mutation produces an exchange relation which is identical in form (but with a different labelling) to the exchange relation at node 2 . We thus obtain a periodic listing of formulae, which can be interpreted as an iteration, as can be seen in the examples below.

\subsection{Period 1 case}

We start with cluster variables $\left(x_{1}, \ldots, x_{N}\right)$, with $x_{i}$ situated at node $i$. We then successively mutate at nodes $1,2,3, \ldots$ and define $x_{N+1}=\tilde{x}_{1}, x_{N+2}=\tilde{x}_{2}$, etc. The exchange relation (17) gives us a formula of the type

$$
x_{n} x_{n+N}=F\left(x_{n+1}, \ldots, x_{n+N-1}\right),
$$

with $F$ being the sum of two monomials. This is interpreted as an $N$ th order recurrence of the real line, with initial conditions $x_{i}=c_{i}$ for $i=1, \ldots, N$. Whilst the right hand side of (18) is polynomial, the formula for $x_{n+N}$ involves a division by $x_{n}$. For a general polynomial $F$, this would mean that $x_{n}$, for $n>2 N$, is a complicated $r a$ tional function of $c_{1}, \ldots, c_{N}$. However, in our case, $F$ is derived through the cluster exchange relation (17), so, by a theorem of [7], $x_{n}$ is just a Laurent polynomial in $c_{1}, \ldots, c_{N}$, for all $n$. In particular, if we start with $c_{i}=1, i=1, \ldots, N$, then $x_{n}$ is an integer for all $n$.

Remark $8.3\left(F \operatorname{not} F_{n}\right)$ For emphasis, we repeat that for a generic quiver we would need to write $F_{n}$, since the formula would be different for each mutation. It is the special property of period 1 quivers which enables the formula to be written as a recurrence. 
The recurrence corresponding to a general quiver of period 1 with $N$ nodes (as described in Theorems $6.1,6.6$ ) corresponding to integers $m_{1}, m_{2}, \ldots, m_{N-1}$ (with $\left.m_{r}=m_{N-r}\right)$ is

$$
x_{n} x_{n+N}=\prod_{\substack{i=1 \\ m_{i}>0}}^{N-1} x_{n+i}^{m_{i}}+\prod_{\substack{i=1 \\ m_{i}<0}}^{N-1} x_{n+i}^{-m_{i}}
$$

Example 8.4 (4 node case) Consider Example 6.3, with $m_{1}=r, m_{2}=-s$, both $r$ and $s$ positive. With $r=1, s=2$, the quiver is shown in Fig. 1(a). We start with the matrix

$$
B(1)=\left(\begin{array}{cccc}
0 & -r & s & -r \\
r & 0 & -r(1+s) & s \\
-s & r(1+s) & 0 & -r \\
r & -s & r & 0
\end{array}\right)
$$

and mutate at node 1 , with $\left(x_{1}, x_{2}, x_{3}, x_{4}\right) \mapsto\left(x_{5}, x_{2}, x_{3}, x_{4}\right)$. Formula (17) gives

$$
x_{1} x_{5}=x_{2}^{r} x_{4}^{r}+x_{3}^{s},
$$

whilst the mutation formula (2) gives

$$
B(2)=\left(\begin{array}{cccc}
0 & r & -s & r \\
-r & 0 & -r & s \\
s & r & 0 & -r(1+s) \\
-r & -s & r(1+s) & 0
\end{array}\right)
$$

Note that the second column of this matrix has the same entries (up to permutation) as the first column of $B(1)$. This is because $\mu_{1} B(1)=\rho B(1) \rho^{-1}$. Therefore, when we mutate $Q(2)$ at node 2 , with $\left(x_{5}, x_{2}, x_{3}, x_{4}\right) \mapsto\left(x_{5}, x_{6}, x_{3}, x_{4}\right)$, formula (17) gives

$$
x_{2} x_{6}=x_{3}^{r} x_{5}^{r}+x_{4}^{s},
$$

which is of the same form as (20), but with indices shifted by 1. Formulae (20) and (21) give us the beginning of the recurrence (18), which now explicitly takes the form

$$
x_{n} x_{n+4}=x_{n+1}^{r} x_{n+3}^{r}+x_{n+2}^{s} .
$$

When $r=1, s=2$, this is exactly the Somos 4 sequence (1). When $r=s=1$, we obtain the recurrence considered by Dana Scott (see [13] and [17]). This case was also considered by Hone (see Theorem 1 in [19]), who showed that it is super-integrable and linearisable. 
Example 8.5 (5 node case) Consider Example 6.4, with $m_{1}=r, m_{2}=-s$, both $r$ and $s$ positive. We start with the matrix

$$
B=\left(\begin{array}{ccccc}
0 & -r & s & s & -r \\
r & 0 & -r(1+s) & -s(r-1) & s \\
-s & r(1+s) & 0 & -r(1+s) & s \\
-s & s(r-1) & r(1+s) & 0 & -r \\
r & -s & -s & r & 0
\end{array}\right)
$$

and mutate at node 1 , with $\left(x_{1}, x_{2}, x_{3}, x_{4}, x_{5}\right) \mapsto\left(x_{6}, x_{2}, x_{3}, x_{4}, x_{5}\right)$. Formula (17) gives

$$
x_{1} x_{6}=x_{2}^{r} x_{5}^{r}+x_{3}^{s} x_{4}^{s} .
$$

Proceeding as before, the general term in the recurrence (18) takes the form

$$
x_{n} x_{n+5}=x_{n+1}^{r} x_{n+4}^{r}+x_{n+2}^{s} x_{n+3}^{s},
$$

which reduces to Somos 5 when $r=s=1$ (giving us the quiver of Fig. 9).

Example 8.6 (6 node case) Consider Example 6.5. The first thing to note is that there are three parameters $m_{i}$, so we have rather more possibilities in our choice of signs. Having already obtained Somos 4 and Somos 5, one may be lured into thinking that Somos 6 will arise. However, Somos 6

$$
x_{n} x_{n+6}=x_{n+1} x_{n+5}+x_{n+2} x_{n+4}+x_{n+3}^{2}
$$

has three terms, so it cannot directly arise through the cluster exchange relation (17), although we remark that it is shown in [8] that the terms in the Somos 6 and Somos 7 sequences are Laurent polynomials in their initial terms. However, various subcases of Somos 6 do arise in our construction. They are, in fact, special cases of the GaleRobinson sequence of Example 8.7.

The case $m_{1}=r, m_{2}=-s, m_{3}=0$ with $r$, s positive We can read off the recurrence from the first column of the matrix of Example 6.5, which is $(0, r,-s, 0,-s, r)^{T}$, giving

$$
x_{n} x_{n+6}=x_{n+1}^{r} x_{n+5}^{r}+x_{n+2}^{s} x_{n+4}^{s},
$$

which gives the first two terms of Somos 6 when $r=s=1$.

The case $m_{1}=r, m_{2}=0, m_{3}=-s$ with $r$, s positive The first column of the matrix is now $(0, r, 0,-s, 0, r)^{T}$, giving

$$
x_{n} x_{n+6}=x_{n+1}^{r} x_{n+5}^{r}+x_{n+3}^{s} .
$$

For a subcase of Somos 6 we choose $r=1, s=2$. 
The case $m_{1}=0, m_{2}=r, m_{3}=-s$ with $r$, s positive The first column of the matrix is now $(0,0, r,-s, r, 0)^{T}$, giving

$$
x_{n} x_{n+6}=x_{n+2}^{r} x_{n+4}^{r}+x_{n+3}^{s},
$$

again with $r=1, s=2$.

Example 8.7 (Gale-Robinson sequence ( $N$ nodes)) The two-term Gale-Robinson recurrence (see (6) of [13]) is given by

$$
x_{n} x_{n+N}=x_{n+N-r} x_{n+r}+x_{n+N-s} x_{n+s},
$$

for $0<r<s \leq N / 2$, and is one of the examples highlighted in [8]. We remark that this corresponds to the period 1 quiver with $m_{r}=1$ and $m_{s}=-1$ (unless $N=2 s$, in which case we take $m_{s}=-2$ ); see Theorem 6.6.

\subsection{Period 2 case}

We start with cluster variables $\left(z_{1}, \ldots, z_{N}\right)$, with $z_{i}$ situated at node $i$. We then successively mutate at nodes $1,2,3, \ldots$ and define $z_{N+1}=\tilde{z}_{1}, z_{N+2}=\tilde{z}_{2}$, etc. However, the exchange relation (17) now gives us an alternating pair of formulae of the type

$$
\begin{aligned}
z_{2 n-1} z_{2 n-1+N} & =F_{0}\left(z_{2 n}, \ldots, z_{2 n+N-2}\right), \\
z_{2 n} z_{2 n+N} & =F_{1}\left(z_{2 n+1}, \ldots, z_{2 n+N-1}\right), \quad n=1,2, \ldots
\end{aligned}
$$

with $F_{i}$ being the sum of two monomials. It is natural, therefore, to relabel the cluster variables as $x_{n}=z_{2 n-1}, y_{n}=z_{2 n}$ and to interpret (22) as a two-dimensional recurrence for $\left(x_{n}, y_{n}\right)$. When $N=2 m$, the recurrence is of order $m$. When $N=2 m-1$, the recurrence is again of order $m$, but the first exchange relation plays the role of a boundary condition. We need $m$ points in the plane to act as initial conditions. When $N=2 m$, the values $z_{1}, \ldots, z_{2 m}$ define these $m$ points. When $N=2 m-1$, we need $z_{2 m}$ in addition to the given initial conditions $z_{1}, \ldots, z_{2 m-1}$. Again, since our recurrences are derived through the cluster exchange relation (17), the formulae for $\left(x_{n}, y_{n}\right)$ are Laurent polynomials of initial conditions. In the case of $N=2 m-1$, this really does mean initial conditions $z_{1}, \ldots, z_{2 m-1}$. The expression for $y_{m}=z_{2 m}$ is already a polynomial, so it is important that it does not occur in the denominators of later terms.

Example 8.8 (4 node case) Consider the general period 2 quiver with four nodes, which has corresponding matrices (10), which we write with $m_{1}=r, m_{2}=-s$, $m_{3}=t$, where $r, s, t$ are positive:

$$
B(1)=\left(\begin{array}{cccc}
0 & -r & s & -t \\
r & 0 & -t-r s & s \\
-s & t+r s & 0 & -r \\
t & -s & r & 0
\end{array}\right),
$$




$$
B(2)=\left(\begin{array}{cccc}
0 & r & -s & t \\
-r & 0 & -t & s \\
s & t & 0 & -r-s t \\
-t & -s & r+s t & 0
\end{array}\right) .
$$

Mutating $Q(1)$ at node 1 , with $\left(z_{1}, z_{2}, z_{3}, z_{4}\right) \mapsto\left(z_{5}, z_{2}, z_{3}, z_{4}\right)$, formula (17) gives

$$
z_{1} z_{5}=z_{2}^{r} z_{4}^{t}+z_{3}^{s},
$$

whilst mutating $Q(2)$ at node 2 , with $\left(z_{5}, z_{2}, z_{3}, z_{4}\right) \mapsto\left(z_{5}, z_{6}, z_{3}, z_{4}\right)$, formula (17) gives

$$
z_{2} z_{6}=z_{3}^{t} z_{5}^{r}+z_{4}^{s}
$$

When $t \neq r$ these formulae are not related by a shift of index. However, since $B(3)=\mu_{2} B(2)=\rho^{2} B(1) \rho^{-2}$, mutating $Q(3)$ at node 3 , with $\left(z_{5}, z_{6}, z_{3}, z_{4}\right) \mapsto$ $\left(z_{5}, z_{6}, z_{7}, z_{4}\right)$, leads to

$$
z_{3} z_{7}=z_{4}^{r} z_{6}^{t}+z_{5}^{s},
$$

which is just (24) with a shift of 2 on the indices. This pattern continues, giving

$$
x_{n} x_{n+2}=y_{n}^{r} y_{n+1}^{t}+x_{n+1}^{s}, \quad y_{n} y_{n+2}=x_{n+1}^{t} x_{n+2}^{r}+y_{n+1}^{s} .
$$

The appearance of $x_{n+2}$ in the definition of $y_{n+2}$ is not a problem, since it can be replaced by the expression given by the first equation.

As shown in Fig. 7, we could equally start with the matrices

$$
\bar{B}(1)=\rho^{-1} B(2) \rho, \quad \bar{B}(2)=\rho B(1) \rho^{-1} .
$$

Since $\bar{B}(1)(r, s, t)=B(1)(t, s, r), \bar{B}(2)(r, s, t)=B(2)(t, s, r)$, we obtain a twodimensional recurrence

$$
u_{n} u_{n+2}=v_{n}^{t} v_{n+1}^{r}+u_{n+1}^{s}, \quad v_{n} v_{n+2}=u_{n+1}^{r} u_{n+2}^{t}+v_{n+1}^{s},
$$

where we have labelled the nodes as $\zeta_{1}, \zeta_{2}, \ldots$ and then substituted $u_{k}=\zeta_{2 k-1}$, $v_{k}=\zeta_{2 k}$. With initial conditions $\left(z_{1}, z_{2}, z_{3}, z_{4}\right)=(1,1,1,1)$ and $\left(\zeta_{1}, \zeta_{2}, \zeta_{3}, \zeta_{4}\right)=$ $(1,1,1,1)$, recurrences $(27)$ and (28) generate different sequences of integers. However, just making the change $\zeta_{4}=2$, reproduces the original $z_{n}$ sequence. This corresponds to a shift in the labelling of the nodes, given by

$$
u_{n}=y_{n}, \quad v_{n}=x_{n+1}, \quad n=1,2, \ldots
$$

Example 8.9 (5 node case) Consider the case with matrices (11), which we write with $m_{1}=r, m_{4}=t$, where $r, t$ are positive. The same procedure leads to the recurrence

$$
\begin{aligned}
y_{n} x_{n+3} & =y_{n+2}^{r} x_{n+1}^{t}+x_{n+2} y_{n+1}, \\
x_{n+1} y_{n+3} & =y_{n+1}^{r} x_{n+3}^{t}+x_{n+2} y_{n+2}, \quad n=1,2, \ldots .
\end{aligned}
$$

together with

$$
x_{1} y_{3}=y_{1}^{r} x_{3}^{t}+x_{2} y_{2},
$$


and initial conditions $\left(x_{1}, y_{1}, x_{2}, y_{2}, x_{3}\right)=\left(c_{1}, c_{2}, c_{3}, c_{4}, c_{5}\right)$. The iteration (29) is a third order two-dimensional recurrence and $y_{3}$ acts as the sixth initial condition.

As above, it is possible to construct a companion recurrence, corresponding to the choice

$$
\bar{B}(1)=\rho^{-1} B(2) \rho, \quad \bar{B}(2)=\rho B(1) \rho^{-1} .
$$

\section{Linearisable recurrences from primitives}

This section is concerned with the recurrences derived from period 1 primitives. Similar results can be shown for higher periods, but we omit these here.

Our primitive quivers are inherently simpler than composite ones (as their name suggests!). The mutation process (at node 1) reduces to a simple matrix conjugation. The cluster exchange relation is still nonlinear, but it turns out to be linearisable, as is shown in this section.

Consider the $k$ th (period 1) primitive $P_{N}^{(k)}$ with $N$ nodes, such as those depicted in Figs. 2 to 4. As before, we attach a variable at each node, labelled $\left(x_{1}, \ldots, x_{N}\right)$, with $x_{i}$ situated at node $i$ for each $i$. We then successively mutate at nodes $1,2,3, \ldots$ and define $x_{N+1}=\tilde{x}_{1}, x_{N+2}=\tilde{x}_{2}$, etc. At the $n$th mutation, we start with the cluster $\left\{x_{n}, x_{n+1}, \ldots, x_{N+n-1}\right\}$. By the periodicity property, the corresponding quiver is always $P_{N}^{(k)}$. The exchange relation (17) gives us the formula

$$
x_{n} x_{n+N}=x_{n+k} x_{n+N-k}+1,
$$

where $x_{n+N}$ is the new cluster variable replacing $x_{n}$. Note that one of the products in (17) is empty. This is the $n$th iteration, which we label $E_{n}$. For $\operatorname{gcd}(k, N)=1$, this is a genuinely new sequence for each $N$. However, when $\operatorname{gcd}(k, N)=m>1$, the sequence (30) decouples into $m$ copies of an iteration of order $(N / m)$.

Specifically, if $N=m s$ and $k=m t$, for integers $s, t$, the quiver $P_{N}^{(k)}$ separates into $m$ disconnected components (see Figs. 2(d), 4(b) and 4(c)). The corresponding sequence decouples into $m$ copies of the sequence associated with the primitive $P_{s}^{(t)}$, since (30) then gives

$$
x_{n} x_{n+m s}=x_{n+m t} x_{n+(s-t) m}+1 .
$$

With $n=m l+r, y_{l}^{(r)}=x_{m l+r}, 0 \leq r \leq m-1$, this gives $m$ identical iteration formulae

$$
y_{l}^{(r)} y_{l+s}^{(r)}=y_{l+t}^{(r)} y_{l+s-t}^{(r)}+1 .
$$

Thus if, in (30), we use the initial conditions $x_{i}=1,1 \leq i \leq N$, we obtain $m$ copies of the integer sequence generated by (31).

\subsection{First integrals}

Subtracting the two equations $E_{n}$ and $E_{n+k}$ (see (30)) leads to

$$
\frac{x_{n}+x_{n+2 k}}{x_{n+k}}=\frac{x_{n+N-k}+x_{n+N+k}}{x_{n+N}} .
$$


With the definition

$$
J_{n, k}=\frac{x_{n}+x_{n+2 k}}{x_{n+k}},
$$

we therefore have

$$
J_{n+N-k, k}=J_{n, k},
$$

giving us $N-k$ independent functions $\left\{J_{i, k}: 1 \leq i \leq N-k\right\}$ (or equivalently $\left\{J_{i, k}\right.$ : $n \leq i \leq n+N-k-1\})$.

Remark 9.1 (Decoupled case) Again, when $\operatorname{gcd}(N, k)=m>1$, the sequence (30) decouples into $m$ copies of (31) and the sequence $J_{n, k}$ (with periodicity $N-k$ ) splits into $m$ copies of the corresponding sequence of $J$ 's for the primitive $P_{s}^{(t)}$ (where $N=m s$ and $k=m t)$, since, putting $n=m l+r$ and $I_{l, t}^{(r)}=J_{m l+r, k}$ we obtain

$$
I_{l, t}^{(r)}=\frac{x_{m l+r}+x_{m l+r+2 m t}}{x_{m l+r+m t}}=\frac{y_{l}^{(r)}+y_{l+2 t}^{(r)}}{y_{l+t}^{(r)}},
$$

satisfying $I_{l+s-t, t}^{(r)}=I_{l, t}^{(r)}$.

Let $\alpha$ be any function of $N-k$ variables and define $\alpha^{(n)}=\alpha\left(J_{n, k}, \ldots\right.$, $J_{n+N-k-1, k}$ ). Then, from the periodicity (33), $\alpha^{(n+N-k)}=\alpha^{(n)}$ (it can happen that the function will have periodicity $r<N-k$ ). Then the function

$$
K_{\alpha}^{(n)}=\sum_{i=0}^{N-k-1} \alpha^{(n+i)}
$$

is a first integral for the recurrence (30), meaning that it satisfies $K_{\alpha}^{(n+1)}=K_{\alpha}^{(n)}$. It is thus always possible to construct, for the recurrence (30), $N-k$ independent first integrals. For $k=1$ this is the maximal number of integrals, unless the recurrence is itself periodic (see [30] for the general theory of integrable maps). by

For example, $N-k$ independent first integrals $\left\{K_{p}^{(n)}: 1 \leq p \leq N-k\right\}$ are given

$$
K_{p}^{(n)}=\sum_{i=0}^{N-k-1} \alpha_{p}^{(n+i)}, \quad \text { where } \alpha_{p}^{(n)}=\prod_{i=0}^{p-1} J_{n+i, k}
$$

Using the condition (33) and the definition (32), it can be seen that the $\alpha_{p}^{(n)}$ depend upon the variables $x_{n}, \ldots, x_{n+N+k-1}$, so (30) must be used to eliminate $x_{n+N}, \ldots, x_{n+N+k-1}$ in order to get the correct form of these integrals in terms of the $N$ independent coordinates.

Example 9.2 As an example, consider the case $N=4$ and $k=1$. This corresponds to the recurrence:

$$
x_{n} x_{n+4}=x_{n+1} x_{n+3}+1
$$


for the primitive $P_{4}^{(1)}$. We have

$$
J_{n, 1}=\frac{x_{n}+x_{n+2}}{x_{n+1}}
$$

Then $\alpha_{1}^{(n)}=J_{n, 1}, \alpha_{2}^{(n)}=J_{n, 1} J_{n+1,1}$ and $\alpha_{3}^{(n)}=J_{n, 1} J_{n+1,1} J_{n+2,1}$. So

$$
\begin{aligned}
K_{1}^{(n)}= & \alpha_{1}^{(n)}+\alpha_{1}^{(n+1)}+\alpha_{1}^{(n+2)}=J_{n, 1}+J_{n+1,1}+J_{n+2,1} ; \\
K_{2}^{(n)}= & \alpha_{2}^{(n)}+\alpha_{2}^{(n+1)}+\alpha_{2}^{(n+2)}=J_{n, 1} J_{n+1,1}+J_{n+1,1} J_{n+2,1}+J_{n+2,1} J_{n+3,1} \\
= & J_{n, 1} J_{n+1,1}+J_{n+1,1} J_{n+2,1}+J_{n+2,1} J_{n, 1} ; \\
K_{3}^{(n)}= & \alpha_{3}^{(n)}+\alpha_{3}^{(n+1)}+\alpha_{3}^{(n+2)}=J_{n, 1} J_{n+1,1} J_{n+2,1}+J_{n+1,1} J_{n+2,1} J_{n+3,1} \\
& +J_{n+2,1} J_{n+3,1} J_{n+4,1} \\
= & 3 J_{n, 1} J_{n+1,1} J_{n+2,1} .
\end{aligned}
$$

Using (34), we obtain $N-k=3$ independent first integrals. For simplicity we write $a=x_{n}, b=x_{n+1}, c=x_{n+2}$ and $d=x_{n+3}$ :

$$
\begin{aligned}
K_{1}^{(n)}= & \frac{a}{b}+\frac{b}{a}+\frac{b}{c}+\frac{c}{b}+\frac{c}{d}+\frac{d}{c}+\frac{1}{a d} ; \\
K_{2}^{(n)}=3 & +\frac{a}{c}+\frac{c}{a}+\frac{b}{d}+\frac{d}{b}+\frac{1}{a c}+\frac{1}{b d}+\frac{a d}{b c}+\frac{b d}{a c}+\frac{a c}{b d}+\frac{b^{2}}{a c} \\
& +\frac{c^{2}}{b d}+\frac{b}{a c d}+\frac{c}{a b d} ; \\
K_{3}^{(n)}= & 3\left(\frac{a}{b}+\frac{b}{a}+\frac{b}{c}+\frac{c}{b}+\frac{c}{d}+\frac{d}{c}+\frac{a}{d}+\frac{d}{a}+\frac{1}{a b}+\frac{1}{b c}+\frac{1}{c d}+\frac{1}{a d}\right) .
\end{aligned}
$$

Remark 9.3 (Decoupled case) Again, when $\operatorname{gcd}(N, k)>1$, the sequence (30) decouples into $m$ copies of (31) and we use the first integrals built out of the functions $I_{l, t}^{(r)}$.

Let the sequence $\left\{x_{n}\right\}$ be given by the iteration (30), with initial conditions $\left\{x_{i}=\right.$ $\left.a_{i}: 1 \leq i \leq N\right\}$. We have $K_{p}^{(n)}=K_{p}^{(1)}$, which is evaluated in terms of $a_{i}$. We also have $\left\{J_{i, k}=c_{i}: 1 \leq i \leq N-k\right\}$, together with the periodicity condition (33), which can also be written as $J_{n, k}=c_{n}$ with $c_{n+N-k}=c_{n}$. The first integrals $K_{p}^{(n)}$ have simpler formulae when written in terms of $c_{1}, \ldots, c_{N-k}$ (each of which is a rational function of the $a_{i}$ ).

Remark 9.4 (Complete integrability) The complete integrability of the maps associated with the $P_{N}^{(1)}(N$ even) is shown in [9].

\subsection{A linear difference equation}

We show in this subsection that the difference equation (30) can be linearised. 
Theorem 9.5 (Linearisation) If the sequence $\left\{x_{n}\right\}$ is given by the iteration (30), with initial conditions $\left\{x_{i}=a_{i}: 1 \leq i \leq N\right\}$, then it also satisfies

$$
x_{n}+x_{n+2 k(N-k)}=S_{N, k} x_{n+k(N-k)},
$$

where $S_{N, k}$ is a function of $c_{1}, \ldots, c_{N-k}$, which is symmetric under cyclic permutations.

Proof of case $k=1$ We first prove this theorem for the case $k=1$, later showing that the general case can be reduced to this.

We fix $k=1$. For $i \in \mathbb{N}$, let $L_{i}=x_{i}+x_{i+2}-c_{i} x_{i+1}$. For $1 \leq i \leq 2 N-3$, we find that $J_{i, 1}=c_{i}$ (see the last paragraph of the previous section), from which it follows that $L_{i}=0$, but we regard the $x_{i}$ as formal variables for the time being (see the end of the proof of Proposition 9.8). For $i=0,1, \ldots, 2 N-2$, we define a sequence $a_{i}$ as follows. Set $a_{0}=0, a_{1}=1$ and then, for $2 \leq n \leq N-1$, define $a_{n}$ recursively by

$$
a_{n}=-a_{n-2}-c_{n-1} a_{n-1} \text {. }
$$

We also set $b_{2 N-2}=0, b_{2 N-3}=1$ and then, for $N-1 \leq n \leq 2 N-3$, define $b_{n}$ recursively by

$$
b_{n}=-b_{n+2}-c_{n+1} b_{n+1} \text {. }
$$

Lemma 9.6 For $0 \leq n \leq N-1$, we have $b_{2 N-2-n}=\left.a_{n}\right|_{c_{l} \mapsto c_{2 N-2-l}}$.

Proof This is easily shown using induction on $n$ and (36) and (37).

The proofs of the following results (Lemma 9.7, Proposition 9.8 and Corollary 9.9) will be given in the appendix. We first describe the $a_{n}$ explicitly. Define

$$
\begin{aligned}
t_{k, \text { odd }}^{n} & =\sum_{\substack{1 \leq i_{1}<i_{2}<\cdots<i_{k} \leq n \\
i_{1} \text { odd }, i_{2} \text { even, }, \cdots}} c_{i_{1}} c_{i_{2}} \cdots c_{i_{k}} \\
t_{k, \text { even }}^{n} & =\sum_{\substack{1 \leq i_{1}<i_{2}<\cdots<i_{k} \leq n \\
i_{1} \text { even, } i_{2} \text { odd }, \ldots}} c_{i_{1}} c_{i_{2}} \cdots c_{i_{k}}
\end{aligned}
$$

Lemma 9.7 Suppose that $0 \leq n \leq N-1$. Then

(a) If $n=2 r$ is even,

$$
a_{2 r}=(-1)^{r} \sum_{k=0}^{r-1}(-1)^{k} t_{2 k+1, \text { odd }}^{(2 r-1)}
$$

(b) If $n=2 r-1$ is odd,

$$
a_{2 r-1}=(-1)^{r-1} \sum_{k=0}^{r-1}(-1)^{k} t_{2 k, \text { odd }}^{(2 r-2)} .
$$


(c) We have $a_{n}=\left.a_{n}\right|_{c_{l} \mapsto c_{n-l}}$ and $a_{N-1}=b_{N-1}$.

For $n \in \mathbb{N}$ and $0 \leq k \leq n$, define

$$
t_{k, \text { alt }}^{n}= \begin{cases}\sum_{\begin{array}{c}
1 \leq i_{1}<i_{2}<\cdots<i_{k} \leq n \\
i_{1}, i_{2}, \ldots, i_{k} \text { of alternating parity }
\end{array}} c_{i_{1}} c_{i_{2}} \cdots c_{i_{k}} & \text { if } k>0 \\
2 & \text { if } k=0 .\end{cases}
$$

Let $L=\sum_{i=1}^{N-1}(-1)^{i} a_{i} L_{i}+\sum_{i=N}^{2 N-3}(-1)^{i} b_{i} L_{i}$. Since $a_{1}=b_{2 N-3}=1$, the coefficients of $x_{1}$ and $x_{2 N-1}$ in $L$ are both 1. By (36) and (37), the coefficient of $x_{i}$ in $L$ is zero for $i=2,3, \ldots, N-1, N+2, \ldots, 2 N-2$. By Lemma 9.7(c), $a_{N-1}=b_{N-1}$, and it follows that the coefficient of $x_{N+1}$ is also zero. The coefficient of $x_{N}$ is

$$
S_{N, 1}=(-1)^{N-2}\left(a_{N-2}+c_{N-1} a_{N-1}+b_{N}\right) .
$$

Note that $b_{N}=\left.a_{N-2}\right|_{c_{l} \mapsto c_{2 N-2-l}}$ by Lemma 9.6, so $b_{N}=\left.a_{N-2}\right|_{c_{l} \mapsto c_{N-1-l}}$, since $c_{n+N-1}=c_{n}$. This allows us to compute the coefficient of $x_{N}$ explicitly to give

Proposition 9.8 We have

$$
x_{1}+x_{2 N-1}=S_{N, 1} x_{N},
$$

where

$$
S_{N, 1}= \begin{cases}(-1)^{r-1} \sum_{k=0}^{r-1}(-1)^{k} t_{2 k+1, \text { alt }}^{(2 r-1)} & \text { if } N=2 r \text { is even; } \\ (-1)^{r-1} \sum_{k=0}^{r-1}(-1)^{k} t_{2 k, \text { alt }}^{(2 r-2)} & \text { if } N=2 r-1 \text { is odd. }\end{cases}
$$

Corollary 9.9 For all $n \in \mathbb{N}$,

$$
x_{n}+x_{n+2(N-1)}=S_{N, 1} x_{n+N-1},
$$

where $S_{N, 1}$ is as above.

Example 9.10 We calculate $S_{N, 1}$ for some small values of $N$. We have

$$
\begin{aligned}
S_{2,1}= & c_{1} ; \\
S_{3,1}= & c_{1} c_{2}-2 ; \\
S_{4,1}= & c_{1} c_{2} c_{3}-c_{1}-c_{2}-c_{3} ; \\
S_{5,1}= & c_{1} c_{2} c_{3} c_{4}-c_{1} c_{2}-c_{2} c_{3}-c_{3} c_{4}-c_{4} c_{1}+2 ; \\
S_{6,1}= & c_{1} c_{2} c_{3} c_{4} c_{5}-c_{1} c_{2} c_{3}-c_{2} c_{3} c_{4}-c_{3} c_{4} c_{5}-c_{4} c_{5} c_{1}-c_{5} c_{1} c_{2}+c_{1}+c_{2} \\
& +c_{3}+c_{4}+c_{5} ; \\
S_{7,1}= & c_{1} c_{2} c_{3} c_{4} c_{5} c_{6}-c_{1} c_{2} c_{3} c_{4}-c_{2} c_{3} c_{4} c_{5}-c_{3} c_{4} c_{5} c_{6}-c_{4} c_{5} c_{6} c_{1}-c_{5} c_{6} c_{1} c_{2} \\
& -c_{6} c_{1} c_{2} c_{3}+c_{1} c_{2}+c_{1} c_{4}+c_{1} c_{6}+c_{3} c_{4}+c_{3} c_{6}+c_{5} c_{6}+c_{2} c_{3} \\
& +c_{2} c_{5}+c_{4} c_{5}-2 .
\end{aligned}
$$


We remark that $N=7$ gives the first example where the terms of fixed degree in $S_{N, 1}$ (in this case degree 2) do not form a single orbit under the cyclic permutation (1 $2 \cdots N-1)$.

The case of $N=4$ can be found in [19].

\subsubsection{The case of general $k>1$}

When $k>1$, the system of equations $J_{n, k}=c_{n}$ (with $c_{n+N-k}=c_{n}$ ) splits into $k$ subsystems. Writing $n=m k-r$ for some $m \geq 1$ and $0 \leq r<k$ we define

$$
z_{m}=x_{m k-r}, \quad \text { and } \quad I_{m, 1}^{(r)}=\frac{z_{m}+z_{m+2}}{z_{m+1}} .
$$

Writing $J_{n, k}$ (see (32)) in terms of $z_{m}$, we see that $J_{n, k}=I_{m, 1}^{(r)}$. Define $M=$ $N-k+1$, so $c_{n+M-1}=c_{n}$. If $\operatorname{gcd}(N, k)=1$, then, for each $r, I_{m, 1}^{(r)}$ cycle through all of $c_{1}, \ldots, c_{M-1}$ (in some order). For $r=k-1$, label this sequence of $c_{i}$ as $d_{1}, \ldots, d_{M-1}$. It is important to note that, for other values of $r$, the order is just a cyclic permutation of $d_{1}, \ldots, d_{M-1}$. We therefore have the conditions for Corollary 9.9, giving

$$
z_{m}+z_{m+2(M-1)}=S_{M, 1}\left(d_{1}, \ldots, d_{M-1}\right) z_{m+(M-1)} .
$$

Writing this in terms of $x_{n}$ gives (35) with $S_{N, k}=S_{M, 1}\left(d_{1}, \ldots, d_{M-1}\right)$, given by Proposition 9.8.

When $(N, k) \neq 1$, we should first use (31) to reduce to the relatively prime case and proceed as above.

Remark 9.11 We need $2 k(N-k)$ initial conditions in order to generate a sequence with (35), but are only supplied with $\left\{x_{i}=a_{i}: 1 \leq i \leq N\right\}$. If we use the iteration (30) to generate the remaining initial conditions for (35), then (30) and (35) will generate exactly the same sequence of numbers.

\subsection{Pell's equation}

For $k=1$, the sequence (30) arising from the primitive $B_{n}^{1}$ has entries which are closely related to Pell's equation, as indicated to us by examples in [28], e.g. sequences A001519 and A001075 for $N=2, N=3$, respectively. By Theorem 9.5, we have

$$
x_{n}+x_{n+2(N-1)}=S_{N, 1} x_{n+N-1},
$$

for $n \geq 1$. We have set $x_{n}=1$ for $1 \leq n \leq N$, and it is easy to check that $x_{n}=$ $n-N+1$ for $N \leq n \leq 2 N-1$. It follows that $S_{N, 1}=N+1$. Subsequences of the form $y_{m}=x_{m(N-1)+c}$ for some constant $c$ satisfy the recurrence $y_{m}+y_{m+2}=$ $(N+1) y_{m+1}$ which has associated quadratic equation $\lambda^{2}-(N+1) \lambda+1=0$, with roots

$$
\alpha_{ \pm}=\frac{N+1 \pm \sqrt{(N+1)^{2}-4}}{2}
$$




\section{Proposition 9.12}

(a) Suppose that $N=2 r-1$ is odd. For $m \in \mathbb{Z}, m \geq 0$, let $a_{m}=x_{(N-1) m+r}$. Choose $1 \leq t \leq N-1$, and let $b_{m}=x_{(N-1) m+t+1}-x_{(N-1) m+t}$. Then the pairs $\left(a_{m}, b_{m}\right)$ for $m>0$ are the positive integer solutions of the Pell equation $a^{2}-\left(r^{2}-1\right) b^{2}=1$.

(b) Suppose that $N=2 r$ is even. Choose $t$, $t^{\prime}$ such that $1 \leq t \leq r$ and $1 \leq t^{\prime} \leq$ $N-1$. For $m \in \mathbb{Z}, m \geq 0$, let $a_{m}=x_{(N-1) m+t}+x_{(N-1) m+N+1-t}$ and let $b_{m}=x_{(N-1) m+t^{\prime}+1}-x_{(N-1) m+t^{\prime}}$. Then the pairs $\left(a_{m}, b_{m}\right)$ for $m>0$ are the positive integer solutions of the Pell equation $a^{2}-\left((2 r+1)^{2}-4\right) b^{2}=4$.

Proof The general solution of $y_{m}+y_{m+2}=(N+1) y_{m+1}$ is

$$
y_{m}=A_{+} \alpha_{+}^{m-1}+A_{-} \alpha_{-}^{m-1}
$$

for arbitrary constants $A_{ \pm}$. The description above of the initial terms in the sequence $\left(x_{n}\right)$ gives initial terms (for $m=0$ and 1) for the subsequences $a_{m}$ and $b_{m}$ in each case, and it follows that, in the odd case,

$$
a_{m}+b_{m} \sqrt{r^{2}-1}=\left(r+\sqrt{r^{2}-1}\right)^{m}
$$

and, in the even case,

$$
a_{m}+b_{m} \sqrt{(2 r+1)^{2}-4}=2^{1-m}\left(2 r+1+\sqrt{(2 r+1)^{2}-4}\right)^{m} .
$$

In the odd case, it is well-known that these are the positive integer solutions to $a^{2}-$ $\left(r^{2}-1\right) b^{2}=1$, and in the even case, the description of the solutions is given in [18] (see also [32, Theorem 1]). (For the $N=2$ case, see for example [28], sequence A001519.)

\section{Parameters and coefficients}

We recalled the definition of a skew-symmetric coefficient-free cluster algebra in Sect. 8. The general definition [7] of a cluster algebra allows for coefficients in the exchange relations. We use the ice quiver approach of $[12,2.2]$ in which some of the cluster variables are specified to be frozen. The definition of the cluster algebra is the same, except that mutation at the frozen cluster variables is not allowed. Our aim in this section is to describe the period 1 ice quivers. Each such quiver models a corresponding Laurent recurrence with parameters, again via the Laurent phenomenon $[7,3.1]$. In other words, we will give an answer to the question as to when can we take an iterative binomial recurrence coming from a periodic quiver and add coefficients to the recurrence and still explain this recurrence in terms of periodic mutations of a frozen quiver using our methods. We will then give some examples of recurrences modelled by periodic ice quivers.

We consider an initial cluster consisting of $N$ unfrozen cluster variables $x_{1}, x_{2}, \ldots$, $x_{N}$ and $M$ frozen cluster variables $y_{1}, y_{2}, \ldots, y_{M}$. Thus, each seed contains a cluster with $N$ unfrozen cluster variables together with the frozen variables $y_{1}, \ldots, y_{M}$, 
which never change. The quiver in the seed has $N$ unfrozen vertices $1,2, \ldots, N$ and $M$ frozen vertices $N+1, \ldots, N+M$. The exchange matrix $B$ will be taken to be the corresponding skew-symmetric matrix. The entries $b_{N+i, N+j}, 1 \leq i, j \leq M$ do not play a role, so we take them to be zero (equivalently, there are no arrows from a vertex $N+1, \ldots, N+M$ of the quiver to another such vertex).

Note that in the usual frozen variable set-up, columns $N+1, \ldots, N+M$ of $B$ are not included. This makes no difference, since the entries in these columns do not appear in the exchange relations. They are determined by the rest of $B$ since $B$ is skew-symmetric, and by the above assumption on zero entries. In order to ensure that the entries $b_{N+i, N+j}$ remain zero, we must modify the mutation $\mu_{i}$ slightly: $\tilde{\mu}_{i}$ is the same as $\mu_{i}$ except that the entries $b_{N+i, N+j}, 1 \leq i, j \leq M$, remain zero by definition. We find it convenient to include the extra columns in order to study the period 1 ice quiver case.

The exchange relation can then be written as follows, for $1 \leq \ell \leq n$ :

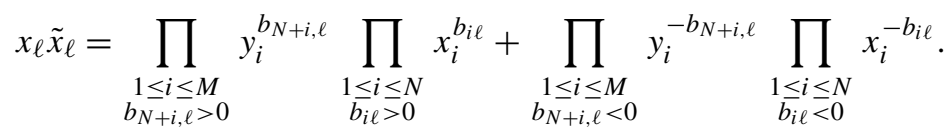

Thus, the coefficients appearing in the exchange relation change with each successive mutation, since they depend on the exchange matrix.

Let $\tilde{\rho}=\left(\begin{array}{cc}\rho & \mathbf{0} \\ \mathbf{0} & I_{M}\end{array}\right)$, where $\mathbf{0}$ denotes zeros and $I_{M}$ denotes the $M \times M$ identity matrix. Thus $\widetilde{\rho}$ represents the permutation sending $(1,2, \ldots, N)$ to $(N, 1,2, \ldots, N-1)$ and fixing $N+1, \ldots, N+M$.

Definition 10.1 A quiver $Q$, with $N+M$ vertices as above, satisfying

$$
\widetilde{\mu_{1}} B_{Q}=\widetilde{\rho} B_{Q} \widetilde{\rho}^{-1}
$$

is said to be a period 1 ice quiver.

Since the effect of conjugation by $\widetilde{\rho}$ on the first $N$ elements of each of the rows $N+1, \ldots, N+M$ of the matrix $B_{Q}$ is to cyclically shift them along one position to the right (with the entries in the opposite positions in the extra columns cyclically moving down one position), it is easy to see that, if we mutate such a quiver $Q$ successively at vertices $1,2, \ldots, N, 1,2, \ldots$ etc. we obtain the terms (as in Sect. 8) of a recurrence:

$$
x_{n} x_{n+N}=F\left(x_{n+1}, \ldots, x_{n+N-1}, y_{1}, y_{2}, \ldots, y_{M}\right)
$$

where $F$ is a sum of two monomials in the $x_{i}$ with coefficients given by fixed monomials in the $y_{i}$. By the Laurent Phenomenon [7, 3.1], each cluster variable can be written as a Laurent polynomial in $x_{1}, x_{2}, \ldots, x_{N}$ with coefficients in $\mathbb{Z}\left[y_{1}, y_{2}, \ldots, y_{M}\right]$. Thus, the recurrence will be Laurent in this sense.

Theorem 10.2 Let $Q$ be an ice quiver on $N+M$ vertices, $1,2, \ldots, N+M$, with vertices $N+1, \ldots, N+M$ frozen. Then $Q$ is a period 1 ice quiver if and only if the 
induced subquiver on vertices $1,2, \ldots, N$ is a period 1 quiver and, if $B_{Q}$ is written as in Theorem 6.1, the following are satisfied.

(a) If $N=2 r+1$ is odd, then for each $1 \leq i \leq M$ such that row $N+i$ of $B$ is nonzero there is $t_{i} \in\{1, \ldots, r\}$ such that $m_{t_{i}}=m_{N-t_{i}}=-1$ and all other $m_{j}$, for $1 \leq j \leq N-1$, are nonnegative, and a positive integer $l_{i}$ such that

$$
b_{N+i, j}= \begin{cases}l_{i}, & 1 \leq j \leq t_{i}, \\ 0, & t_{i}+1 \leq j \leq 2 r+1-t_{i}, \\ -l_{i}, & 2 r+2-t_{i} \leq j \leq 2 r+1=N, \\ 0, & N+1 \leq j \leq N+M .\end{cases}
$$

Alternatively, the $m_{j}$ are as above with the opposite signs and the entries in the row are the negative of the above.

(b) If $N=2 r$ is even, then for each $1 \leq i \leq M$ such that row $N+i$ of $B$ is non-zero there is $t_{i} \in\{1, \ldots, r-1\}$ such that $m_{t_{i}}=m_{N-t_{i}}=-1$ and the other $m_{j}$, for $1 \leq j \leq N-1$, are nonnegative, or $m_{r}=-2$ and all other $m_{j}$ are nonnegative. Furthermore, there is a positive integer $l_{i}$ such that

$$
b_{N+i, j}= \begin{cases}l_{i}, & 1 \leq j \leq t_{i}, \\ 0, & t_{i}+1 \leq j \leq 2 r-t_{i}, \\ -l_{i}, & 2 r+1-t_{i} \leq j \leq 2 r=N, \\ 0, & N+1 \leq j \leq N+M .\end{cases}
$$

Alternatively, the $m_{j}$ are as above with the opposite signs and the entries in the row are the negative of the above.

Proof To solve (41), it is clear that the induced subquiver of $Q$ on vertices $1,2, \ldots, N$ must be a period 1 quiver in our usual sense. So we assume that the entries $b_{i j}$ for $1 \leq i, j \leq N$ are as in the general solution given in Theorem 6.1. For $1 \leq i \leq M$, the $N+i, j$ entry of $\widetilde{\rho} B_{Q} \widetilde{\rho}^{-1}$ is $b_{N+i, j-1}$ (where $j-1$ is read as $N$ if $j=1$ ). Thus we must solve the equations $b_{N+i, N}=-b_{N+i, 1}$ and $b_{N+i, j-1}=b_{N+i, j}+$ $\frac{1}{2}\left(b_{N+i, 1}\left|b_{1, j}\right|+\left|b_{N+i, 1}\right| b_{1, j}\right)$ for $i=1,2, \ldots, M$ and $j=2, \ldots, N$, noting that columns $N+1, \ldots, N+M$ will give rise to the same equations and that $b_{N+i, j}=0$ for $j>N$.

We thus must solve the equations $b_{N+i, N}+b_{N+i, 1}=0$ and $b_{N+i, j-1}-b_{N+i, j}=$ $\varepsilon\left(b_{N+i, 1}, m_{j-1}\right)$ for $j=2, \ldots, N$. Adding all of these, we obtain the constraint that

$$
2 b_{N+i, 1}=\sum_{j=1}^{N-1} \varepsilon\left(b_{N+i, 1}, m_{j}\right) \text {. }
$$

The solutions are given by the values of $b_{N+i, 1}$ satisfying this constraint (using the other equations to write down the values of the other $b_{N+i, j}$ ).

If $N=2 r+1$ is odd, the constraint can be rewritten

$$
2 b_{N+i, 1}=b_{N+i, 1}\left(\left|m_{1}\right|+\cdots+\left|m_{r}\right|\right)-\left|b_{N+i, 1}\right|\left(m_{1}+\cdots+m_{r}\right),
$$


using the fact that $m_{j}=m_{N-j}$ for all $j$. If $b_{N+i, 1}=0$ then $b_{N+i, j}=0$ for all $j$, so for a non-zero $(N+i)$ th row we must have $b_{N+i, 1} \neq 0$. For solutions with $b_{N+i, 1}>0$, we must have

$$
\left|m_{1}\right|-m_{1}+\left|m_{2}\right|-m_{2}+\cdots+\left|m_{r}\right|-m_{r}=2 .
$$

Since $|x|-x=0$ for $x \geq 0$ and equals $-2 x$ for $x \leq 0$, the only solutions arise when $m_{t_{i}}=-1$ for some $t_{i}$ (so $m_{N-t_{i}}=-1$ also) and all other $m_{j}$ are nonnegative. They are of the form

$$
b_{N+i, j}= \begin{cases}b_{N+i, 1}, & 1 \leq j \leq t_{i} \\ 0, & t_{i}+1 \leq j \leq 2 r+1-t_{i}, \\ -b_{N+i, 1}, & 2 r+2-t_{i} \leq j \leq 2 r+1,\end{cases}
$$

as required. The solutions with negative $b_{N+i, 1}$ are the negative of these (provided $m_{t_{i}}=m_{N-t_{i}}=1$ and all other $m_{j}$ are nonpositive).

If $N=2 r$ is even, the constraint can be rewritten

$2 b_{N+i, 1}=b_{N+i, 1}\left(\left|m_{1}\right|+\cdots+\left|m_{r-1}\right|+\left|m_{r}\right| / 2\right)-\left|b_{N+i, 1}\right|\left(m_{1}+\cdots+m_{r-1}+m_{r} / 2\right)$,

again using the fact that $m_{j}=m_{N-j}$ for all $j$. As in the odd case, we must have $b_{N+i, 1} \neq 0$ for a non-zero $(N+i)$ th row. If $b_{N+i, 1}>0$, we must have

$$
\left|m_{1}\right|-m_{1}+\left|m_{2}\right|-m_{2}+\cdots+\left|m_{r-1}\right|-m_{r-1}+\left|m_{r}\right| / 2-m_{r} / 2=2 .
$$

Arguing as in the odd case, we see that solutions arise when $m_{t_{i}}=-1$ for some $t_{i}$ with $1 \leq t_{i} \leq r-1$ (and so $m_{N-t_{i}}=-1$ ) and all other $m_{j}$ are nonnegative, or when $m_{r}=-2$ and all other $m_{j}$ are nonnegative. They are of the form

$$
b_{N+i, j}= \begin{cases}b_{N+i, 1}, & 1 \leq j \leq t_{i} \\ 0, & t_{i}+1 \leq j \leq 2 r-t_{i} \\ -b_{N+i, 1}, & 2 r+1-t_{i} \leq j \leq 2 r\end{cases}
$$

where $t_{i}=r$ for the last case, as required. The solutions for negative $b_{N+i, 1}$ are the negative of these (with the negative of the constraints on the $m_{j}$ ).

Corollary 10.3 Consider the Laurent recurrence (19) corresponding to a period 1 quiver:

$$
x_{n} x_{n+N}=\prod_{\substack{i=1 \\ m_{i}>0}}^{N-1} x_{n+i}^{m_{i}}+\prod_{\substack{i=1 \\ m_{i}<0}}^{N-1} x_{n+i}^{-m_{i}} .
$$

The same recurrence, with parameters introduced on the right hand side as coefficients of the monomials, arises from a period 1 ice quiver as above if and only if a parameter on a monomial is only allowed when the other monomial is of the form $x_{n+i} x_{n+N-i}$ for some $i$ with $1 \leq i \leq N / 2$. (If both monomials are of this form then a parameter is allowed on both.) If this condition is satisfied, the recurrence with parameters is again Laurent. 
Fig. 10 The ice quiver for

Somos 4 with parameters

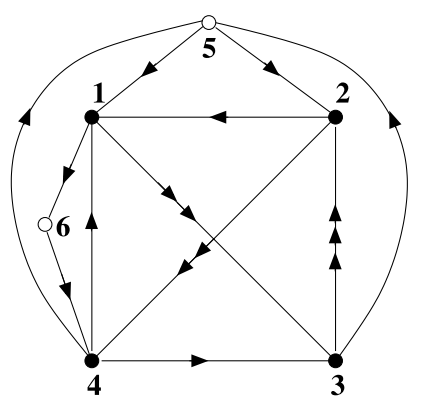

This corollary has the following interesting consequence:

Proposition 10.4 (Gale-Robinson recurrence) The only binomial recurrences corresponding to period 1 quivers that, when parameters on both monomials are allowed, correspond to period 1 ice quivers, are the two-term Gale-Robinson recurrences.

Proof The Gale-Robinson recurrences are exactly those for which both monomials are of the required form in Corollary 10.3. See Example 8.7 in Sect. 8.1.

Note that it follows that these recurrences, with a parameter multiplying each of the monomials, are Laurent. This was shown in [8, 1.7].

Example 10.5 (Somos 4 recurrence with parameters) The Somos 4 recurrence is a special case of the two-term Gale-Robinson recurrence. We can add extra coefficient rows $(1,1,-1,-1,0,0)$ and $(-1,0,0,1,0,0)$ to the corresponding matrix, giving the six-vertex quiver shown in Fig. 10, with empty circles denoting frozen vertices. We recover the Laurent property of the corresponding recurrence:

$$
x_{n+4} x_{n}=y_{1} x_{n+1} x_{n+3}+y_{2} x_{n+2}^{2} .
$$

Example 10.6 (A recurrence considered by Dana Scott) We have seen (see Example 8.4 of Sect. 8.1) that the 4-node case $m_{1}=1, m_{2}=-1$ corresponds to a recurrence considered by Dana Scott. By Theorem 10.2(b) the only possible non-zero extra row in the matrix is $(-1,0,0,1,0,0)$ (or a positive multiple) giving the recurrence:

$$
x_{n+4} x_{n}=x_{n+1} x_{n+3}+y x_{n+2} \text {. }
$$

It follows that this recurrence is Laurent. The corollary, that this recurrence gives integers for all integer $y$ (if $x_{1}=x_{2}=x_{3}=x_{4}=1$ ), was noted in [13]. It is also noted in [13] that the recurrence

$$
x_{n+4} x_{n}=2 x_{n+1} x_{n+3}+x_{n+2}
$$

(with the same initial conditions) does not give integers. It follows that the recurrence

$$
x_{n+4} x_{n}=y_{1} x_{n+1} x_{n+3}+x_{n+2}
$$

does not have the Laurent property. 
Remark 10.7 (A conjecture) Although our results do not determine non-Laurentness it is interesting to note that we obtain exactly those which are Laurent as solutions in the above examples. It seems reasonable to conjecture that the parameter versions of recurrences arising from period 1 quivers are Laurent if and only if they arise from a cluster algebra with frozen variables in the above sense.

\section{Supersymmetric quiver gauge theories}

In this section we point to the D-brane literature in which our quivers arise in the context of quiver gauge theories. The quivers arising in supersymmetric quiver gauge theories often have periodicity properties. Indeed, in [24, §3] the authors consider an $\mathcal{N}=1$ supersymmetric quiver gauge theory associated to the complex cone over the second del Pezzo surface $d P_{2}$. The quiver $Q_{d P_{2}}$ of the gauge theory they consider is given in Fig. 11(d). The authors compute the Seiberg dual of the quiver gauge theory at each of the nodes of the quiver. The Seiberg dual theory has a new quiver, obtained using a combinatorial rule from the original quiver using the choice of vertex (see [6]). It can be checked that the combinatorial rule for Seiberg dualising a quiver coincides with the rule for Fomin-Zelevinsky quiver mutation (Definition 2.1); see [31] for a discussion of the relationship between Seiberg duality and quiver mutation. In [24, §3] the authors compute the Seiberg dual of $Q_{d P_{2}}$ at each node, in particular showing that the Seiberg dual of $Q_{d P_{2}}$ at node 1 is an isomorphic quiver. They indicate that such behaviour is to be expected from a physical perspective.

This quiver fits into the scheme discussed in this article: it is a period 1 quiver. In fact it coincides with the quiver corresponding to the matrix $B_{5}^{(1)}-B_{5}^{(2)}+B_{3}^{(1)}$ (see Example 6.4 with $m_{1}=1, m_{2}=-1$, and also Fig. 9), with the relabelling $(1,2,3,4,5) \mapsto(3,4,5,2,1)$ (we give all relabellings starting from our labels). We note that this quiver also appears in [26] (with a relabelling $(1,2,3,4,5) \mapsto$ $\left.\left(2^{\prime}, 3^{\prime}, 1,2,3\right)\right)$ in the context of a $d P_{2}$ brane tiling and that the corresponding sequence is the Somos 5 sequence.

The quivers of quiver gauge theories associated to the complex cones over the Hirzebruch zero and del Pezzo 0-3 surfaces are computed in [5, §4]. We list them for convenience in Fig. 11 for the Hirzebruch 0 and del Pezzo 0,1 and 3 surfaces. Note that the del Pezzo 2 case was discussed above: we chose the quiver given in $[24, \S 3]$ for this case because it fits better into our set-up. We remark that the quiver for $d P_{1}$ coincides with the Somos 4 quiver (with matrix $B_{4}^{(1)}-2 B_{4}^{(2)}+2 B_{2}^{(1)}$ ), with

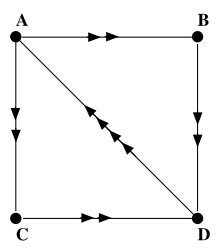

(a) Hirzebruch 0

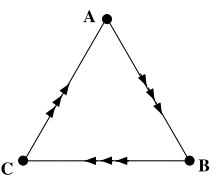

(b) del Pezzo 0

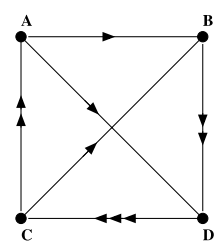

(c) del Pezzo 1

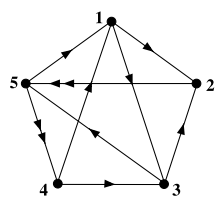

(d) del Pezzo 2

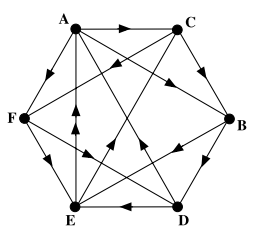

(e) del Pezzo 3

Fig. 11 Quivers of quiver gauge theories associated to a family of surfaces. Quiver (d) is from [24] while the others are from [5] 
the relabelling $(1,2,3,4) \mapsto(B, C, D, A)$. Thus it is period 1. See Example 6.3 and Fig. 1(a).

The quiver for the Hirzebruch 0 surface is period 2: with the relabelling $(1,2,3,4) \mapsto(C, A, D, B)$. It corresponds to the matrix $B(1)$ given in (10) in Sect. 7.2 with $m_{1}=2, m_{2}=-2$ and $m_{3}=0$. The quiver for $d P_{3}$ is period two. In fact it is one of the period two quivers described in Sect. 7.4, with $m_{1}=m_{3}=1$, $m_{2}=-1$ and $m_{\overline{1}}=0$.

Finally, we note that, by construction, the in-degree of a vertex $i$ always coincides with the out-degree of $i$ for any quiver arising from a brane tiling in the sense of [11]. It is interesting to note that the only quivers of cluster mutation period 1 satisfying this assumption with five or fewer vertices are the Somos 4 and Somos 5 quivers, i.e. quivers associated to $d P_{1}$ and $d P_{2}$ (see Figs. 11(c) and 11(d)).

\section{Conclusions}

In this paper we have raised the problem of classifying all quivers with mutation periodicity. For period 1 we have given a complete solution. For period 2 we have given a solution which exists for all $N$ (the number of nodes). In addition to these, we have seen that for $N=5$ there are some "exceptional solutions", such as (12) and (13). We conjecture that such "exceptional solutions" will exist for all odd $N$, but not for the even case. We also conjecture that there are more general infinite families than the one presented in Sect. 7.4, where we took a particularly simple initial condition for the iteration (16). We could, for instance, consider the case with $m_{N-r}=m_{\bar{r}}$ for several values of $r$. We could also consider a family of period $r$ quivers, satisfying $\mu_{1}(B)=\rho B(\sigma(\mathbf{m})) \rho^{-1}$, with $\sigma^{r}(\mathbf{m})=\mathbf{m}$.

The other main theme of our paper was the construction and analysis of recurrences with the Laurent property. We have shown that the recurrences associated with our period 1 primitives can be linearised. This construction can be extended to higher period cases, but we currently only have examples. General periodic quivers give rise to truly nonlinear maps, the simplest of which is Somos 4, which is known [19] to be integrable and, in fact, related to the QRT [27] map of the plane. Somos 5 is similarly known to be integrable, as are the subcases of Somos 6 discussed in Example 8.6. On the other hand,

$$
x_{n} x_{n+6}=x_{n+1}^{2} x_{n+5}^{2}+x_{n+2}^{2} x_{n+3}^{4} x_{n+4}^{2},
$$

(corresponding to the choice $m_{1}=-m_{2}=2, m_{3}=-4$ in Example 8.6) is known to be not integrable (see (4.3) of [19]). Even though this recurrence has the Laurent property and satisfies "singularity confinement" [15] (a type of Painlevé property for discrete equations), it fails the more stringent "algebraic entropy" [3] test for integrability. This simple test (or the related "diophantine integrability" [16] test) can very quickly show that a map is not integrable. If they indicate integrability, then it is sensible to search for invariant functions in order to prove integrability. Early indications (preliminary calculations by C-M Viallet) are that for integers $m_{i}$ which are "small in absolute value" we have integrable cases. We thus expect that small sub-families of our general periodic quivers will give rise to integrable maps. The 
isolation and classification of integrable cases of the recurrences discussed in this article is an important open question, which will be discussed in [10].

We have seen that many of our examples occur in the context of supersymmetric quiver gauge theories. A deep understanding of the connection with brane tilings and related topics would be very interesting.

After this paper first appeared on the arXiv, Jan Stienstra pointed out to us that the quivers in Fig. 11 also appear in [29] in the context of Gelfand-Kapranov-Zelevinsky hypergeometric systems in two variables, suggesting a possible connection between cluster mutation and such systems.

Since we wrote the first version, the article [25] has appeared, proposing a general study of periodicity in cluster algebras (in a wide sense), motivated by many interesting examples of periodicity for cluster algebras and $T$ and $Y$ systems (see references therein). In particular, periodicity of (seeds in) cluster algebras plays a key role in the context of the periodicity conjecture for $Y$ systems, which was proved in full generality in [21]; see also references therein.

The articles $[2,22]$ have also appeared, proving the linearisation of frieze sequences (or frises) associated to acyclic quivers (quivers without an oriented cycle); see these two papers and references therein for more details of these sequences. If $Q$ is acyclic, its vertices $\{1,2, \ldots, N\}$ can be numbered so that $i$ is a sink on the induced subquiver on vertices $i, i+1, \ldots, N$ for each $i$; then $1,2, \ldots, N$ is an admissible sequence of sinks in the sense of Remark 2.4 and so $Q$ has period $N$ in our sense. The corresponding sequence of cluster variables can be regarded as an $N$-dimensional recurrence (as in the period 2 case, 8.2). Generalising results of [2], in [22] it is shown that all components of this recurrence are linearisable if $Q$ is Dynkin or affine (and conversely; in fact the result is more general, including valued quivers). This linearisability can be regarded as a generalisation of Theorem 9.5, since our primitives are acyclic and the numbering of their vertices satisfies the above sink requirement, using Lemma 4.4. In fact this last statement is true for primitives of any period, so it follows from [22] that the recurrences corresponding to primitives of any period are linearisable, noting that the period of a primitive always divides $N$.

Acknowledgements We would like to thank Andy Hone for some helpful comments on an earlier version of this manuscript, Jeanne Scott for some helpful discussions, and Claude Viallet for computing the algebraic entropy of some of our maps. We'd also like to thank the referees for their helpful and interesting comments on the submitted version of this article. This work was supported by the Engineering and Physical Sciences Research Council [grant numbers EP/C01040X/2 and EP/G007497/1].

\section{Appendix: Proofs of results in Sect. 9.2}

Proof of Lemma 9.7 We first prove (a) and (b). The result is clearly true for $n=0,1$. Assume that it holds for smaller $n$ and firstly assume that $n=2 r-1$ is odd. Then

$$
\begin{aligned}
a_{2 r-1} & =-a_{2 r-3}-c_{2 r-2} a_{2 r-2} \\
& =(-1)^{r-1} \sum_{k=0}^{r-2}(-1)^{k} t_{2 k, \text { odd }}^{(2 r-4)}+c_{2 r-2}(-1)^{r} \sum_{k=0}^{r-2}(-1)^{k} t_{2 k+1, \text { odd }}^{(2 r-3)}
\end{aligned}
$$




$$
\begin{aligned}
= & (-1)^{r-1} t_{0, \text { odd }}^{(2 r-4)}+(-1)^{r-1} \sum_{k=0}^{r-3}(-1)^{k+1} t_{2 k+2, \text { odd }}^{(2 r-4)} \\
& +c_{2 r-2}(-1)^{r} \sum_{k=0}^{r-2}(-1)^{k} t_{2 k+1, \text { odd }}^{(2 r-3)} \\
= & (-1)^{r-1} t_{0, \text { odd }}^{(2 r-4)}+(-1)^{2 r-2} c_{2 r-2} t_{2 r-3, \text { odd }}^{(2 r-3)} \\
& +(-1)^{r} \sum_{k=0}^{r-3}(-1)^{k}\left(t_{2 k+2, \text { odd }}^{(2 r-4)}+c_{2 r-2} t_{2 k+1, \text { odd }}^{(2 r-3)}\right) \\
= & (-1)^{r-1} t_{0, \text { odd }}^{(2 r-4)}+(-1)^{2 r-2} t_{2 r-2, \text { odd }}^{(2 r-2)}+(-1)^{r} \sum_{k=0}^{r-3}(-1)^{k} t_{2 k+2, \text { odd }}^{(2 r-2)} \\
= & (-1)^{r-1} \sum_{k=0}^{r-1}(-1)^{k} t_{2 k, \text { odd }}^{(2 r-2)},
\end{aligned}
$$

and the result holds for $n$. A similar argument shows that the result holds for $n$ when $n$ is even. Then (a) and (b) follow by induction. To prove (c), we note that $t_{2 k+1 \text {,odd }}^{(2 r-1)}$ is invariant under the transformation $c_{l} \mapsto c_{2 r-l}$ and that $t_{2 k \text {,odd }}^{(2 r-2)}$ is invariant under $c_{l} \mapsto c_{2 r-1-l}$. We then have

$$
b_{N-1}=\left.a_{N-1}\right|_{c_{l} \mapsto c_{2 N-2-l}}=\left.a_{N-1}\right|_{c_{l} \mapsto c_{N-1-l}}=a_{N-1}
$$

using Lemma 9.6 and the fact that $c_{n+N-1}=c_{n}$.

Proof of Proposition 9.8 We first assume that $N=2 r-1$ is odd, so $(-1)^{N-2}=-1$. Then

$$
\begin{aligned}
S_{N, 1}= & -\left(a_{2 r-3}+\left.a_{2 r-3}\right|_{c_{l} \mapsto 2 r-2-l}+c_{2 r-2} a_{2 r-2}\right) \\
= & (-1)^{r-1} \sum_{k=0}^{r-2}(-1)^{k} t_{2 k, \text { odd }}^{(2 r-4)}+(-1)^{r-1} \sum_{k=0}^{r-2}(-1)^{k} t_{2 k, \text { even }}^{(2 r-3)} \\
& +c_{2 r-2}(-1)^{r} \sum_{k=0}^{r-2}(-1)^{k} t_{2 k+1, \text { odd }}^{(2 r-3)} \\
= & (-1)^{r-1} t_{0, \text { odd }}^{(2 r-4)}+(-1)^{r-1} \sum_{k=0}^{r-3}(-1)^{k+1} t_{2 k+2, \text { odd }}^{(2 r-4)}+(-1)^{r-1} \sum_{k=0}^{r-2}(-1)^{k} t_{2 k, \text { even }}^{(2 r-3)} \\
& +c_{2 r-2}(-1)^{r} \sum_{k=0}^{r-2}(-1)^{k} t_{2 k+1, \text { odd }}^{(2 r-3)} \\
= & (-1)^{r-1} t_{0, \text { odd }}^{(2 r-4)}+c_{2 r-2}(-1)^{2 r-2} t_{2 r-3, \text { odd }}^{(2 r-3)}
\end{aligned}
$$




$$
\begin{aligned}
& +(-1)^{r} \sum_{k=0}^{r-3}(-1)^{k}\left(t_{2 k+2, \text { odd }}^{(2 r-4)}+c_{2 r-2} t_{2 k+1, \text { odd }}^{(2 r-3)}\right) \\
& +(-1)^{r-1} \sum_{k=0}^{r-2}(-1)^{k} t_{2 k, \text { even }}^{(2 r-3)} \\
& =(-1)^{r-1} t_{0, \text { odd }}^{(2 r-2)}+(-1)^{2 r-2} t_{2 r-2, \text { odd }}^{(2 r-2)}+(-1)^{r} \sum_{k=0}^{r-3}(-1)^{k} t_{2 k+2, \text { odd }}^{(2 r-2)} \\
& +(-1)^{r-1} \sum_{k=0}^{r-2}(-1)^{k} t_{2 k, \text { even }}^{(2 r-3)} \\
& =(-1)^{r-1} \sum_{k=0}^{r-1}(-1)^{k} t_{2 k, \text { odd }}^{(2 r-2)}+(-1)^{r-1} \sum_{k=0}^{r-2}(-1)^{k} t_{2 k, \text { even }}^{(2 r-2)} \\
& =(-1)^{r-1} \sum_{k=0}^{r-1}(-1)^{k} t_{2 k, \text { alt }}^{(2 r-2)},
\end{aligned}
$$

as required. A similar argument shows that the result holds when $N$ is even.

As we have already observed, for the sequence $\left(x_{i}\right)$ we are interested in, the $L_{i}$ vanish. It follows that $L$ vanishes and we are done.

Proof of Corollary 9.9 The proof of Proposition 9.8 also shows that $x_{2}+x_{2+2 N-1}=$ $\left.S_{N, 1}\right|_{c_{l} \mapsto c_{l+1}} x_{2+N-1}$. It follows from the description of $S_{N, 1}$ in Proposition 9.8 that $S_{N, 1}=\left.S_{N, 1}\right|_{c_{l} \mapsto c_{l+1}}$ (using the fact that $c_{n+N-1}=c_{n}$ ) so we are done for $n=2$. Repeated application of this argument gives the result for arbitrary $n$.

\section{References}

1. Assem, I., Simson, D., Skowroski, A.: Elements of the Representation Theory of Associative Algebras, vol. 1. Techniques of Representation Theory. London Mathematical Society Student Texts, vol. 65. Cambridge University Press, Cambridge (2006)

2. Assem, I., Reutenauer, C., Smith, D.: Frises. Preprint (2009). arXiv:0906.2026v1 [math.RA]

3. Bellon, M., Viallet, C.-M.: Algebraic entropy. Commun. Math. Phys. 204, 425-437 (1999)

4. Derksen, H., Weyman, J., Zelevinsky, A.: Quivers with potentials and their representations I: Mutations. Sel. Math., New Ser. 14, 59-119 (2008)

5. Feng, B., Hanany, A., He, Y.-H.: D-brane gauge theories from toric singularities and toric duality. Nucl. Phys. B 595, 165-200 (2001)

6. Feng, B., Hanany, A., He, Y.-H., Uranga, A.M.: Toric duality as Seiberg duality and brane diamonds. J. High Energy Phys. 12, 035 (2001)

7. Fomin, S., Zelevinsky, A.: Cluster algebras I: foundations. J. Am. Math. Soc. 15, 497-529 (2002)

8. Fomin, S., Zelevinsky, A.: The Laurent phenomenon. Adv. Appl. Math. 28, 119-144 (2002)

9. Fordy, A.P.: Mutation-periodic quivers, integrable maps and associated Poisson algebras. Preprint (2010). arXiv:1003.3952v1 [nlin.SI]

10. Fordy, A.P., Hone, A.N.W.: Integrable maps and Poisson algebras derived from cluster algebras. In preparation

11. Franco, S., Hanany, A., Kennaway, K.D., Vegh, D., Wecht, B.: Brane dimers and quiver gauge theories. J. High Energy Phys. 01, 096 (2006) 
12. Fu, C., Keller, B.: On cluster algebras with coefficients and 2-Calabi-Yau categories. Trans. Am. Math. Soc. 362, 859-895 (2010)

13. Gale, D.: The strange and surprising saga of the Somos sequences. Math. Intell. 13, 40-42 (1991)

14. Gekhtman, M., Shapiro, M., Vainshtein, A.: Cluster algebras and Poisson geometry. Mosc. Math. J. 3, 899-934 (2003)

15. Grammaticos, B., Ramani, A., Papageorgiou, V.G.: Do integrable mappings have the Painlevé property? Phys. Rev. Lett. 67, 1825-1828 (1991)

16. Halburd, R.G.: Diophantine integrability. J. Phys. A 38, L263-L269 (2005)

17. Heideman, P., Hogan, E.: A new family of Somos-like recurrences. Electron. J. Comb. 15, R54 (2008)

18. Hoggatt, V.E., Bicknell-Johnson, M.: A primer for the Fibonacci numbers XVII: generalized Fibonacci numbers satisfying $u_{n+1} u_{n-1}-u_{n}^{2}= \pm 1$. Fibonacci Q. 2, 130-137 (1978)

19. Hone, A.N.W.: Laurent polynomials and superintegrable maps. SIGMA 3, 022 (2007), 18 pages

20. Kashiwara, M.: On crystal bases of the q-analogue of universal enveloping algebras. Duke Math. J. 63, 465-516 (1991)

21. Keller, B.: The periodicity conjecture for pairs of Dynkin diagrams. Preprint (2010). arXiv: 1001.1531v3 [math.RT]

22. Keller, B., Scherotzke, S.: Linear recurrence relations for cluster variables of affine quivers. Preprint (2010). arXiv:1004.0613v2 [math.RT]

23. Lusztig, G.: Canonical bases arising from quantized enveloping algebras. J. Am. Math. Soc. 3, 447498 (1990)

24. Mukhopadhyay, S., Ray, K.: Seiberg duality as derived equivalence for some quiver gauge theories. J. High Energy Phys. 02, 070 (2004)

25. Nakanishi, T.: Periodic cluster algebras and dilogarithm identities. Preprint (2010). arXiv: 1006.0632v3 [math.QA]

26. Oota, T., Yasui, Y.: New example of infinite family of quiver gauge theories. Nucl. Phys. B 762, 377-391 (2007)

27. Quispel, G.R.W., Roberts, J.A.G., Thompson, C.J.: Integrable mappings and soliton equations. Phys. Lett. A 126, 419-421 (1988)

28. Sloane, N.J.A.: The on-line encyclopedia of integer sequences. www.research.att.com/njas/sequences (2009)

29. Stienstra, J.: Hypergeometric systems in two variables, quivers, dimers and dessins d'enfants. In: Yui, N., Verrill, H., Doran, C.F. (eds.) Modular Forms and String Duality. Fields Inst. Commun., vol. 54, pp. 125-161. Am. Math. Soc., Providence (2008)

30. Veselov, A.P.: Integrable maps. Russ. Math. Surv. 46(5), 1-51 (1991)

31. Vitoria, J.: Mutations vs. Seiberg duality. J. Algebra 321(3), 816-828 (2009)

32. Zay, B.: An application of the continued fractions for $\sqrt{D}$ in solving some types of Pell's equations. Acta Acad. Paedagog. Agriensis Sect. Mat. (NS) 25, 3-14 (1998) 